\title{
On a generalization of the Selection Theorem of Mahler
}

\author{
par GiLBert MURAZ et JEAN-Louis VERGER-GAUGRY
}

RÉSUMÉ. On montre que l'ensemble $\mathcal{U} \mathcal{D}_{r}$ des ensembles de points de $\mathbb{R}^{n}, n \geq 1$, qui ont la propriété que leur distance interpoint minimale est plus grande qu'une constante strictement positive $r>0$ donnée est muni d'une métrique pour lequel il est compact et tel que la métrique de Hausdorff sur le sous-ensemble $\mathcal{U} \mathcal{D}_{r, f} \subset \mathcal{U} \mathcal{D}_{r}$ des ensembles de points finis est compatible avec la restriction de cette topologie à $\mathcal{U} \mathcal{D}_{r, f}$. Nous montrons que ses ensembles de Delaunay (Delone) de constantes données dans $\mathbb{R}^{n}, n \geq 1$, sont compacts. Trois (classes de) métriques, dont l'une de nature cristallographique, nécessitant un point base dans l'espace ambiant, sont données avec leurs propriétés, pour lesquelles nous montrons qu'elles sont topologiquement équivalentes. On prouve que le processus d'enlèvement de points est uniformément continu à l'infini. Nous montrons que ce Théorème de compacité implique le Théorème classique de Sélection de Mahler. Nous discutons la généralisation de ce résultat à des espaces ambiants autres que $\mathbb{R}^{n}$. L'espace $\mathcal{U} \mathcal{D}_{r}$ est l'espace des empilements de sphères égales de rayon $r / 2$.

ABSTRACT. The set $\mathcal{U} \mathcal{D}_{r}$ of point sets of $\mathbb{R}^{n}, n \geq 1$, having the property that their minimal interpoint distance is greater than a given strictly positive constant $r>0$ is shown to be equippable by a metric for which it is a compact topological space and such that the Hausdorff metric on the subset $\mathcal{U} \mathcal{D}_{r, f} \subset \mathcal{U} \mathcal{D}_{r}$ of the finite point sets is compatible with the restriction of this topology to $\mathcal{U} \mathcal{D}_{r, f}$. We show that its subsets of Delone sets of given constants in $\mathbb{R}^{n}, n \geq 1$, are compact. Three (classes of) metrics, whose one of crystallographic nature, requiring a base point in the ambient space, are given with their corresponding properties, for which we show topological equivalence. The point-removal process is proved to be uniformly continuous at infinity. We prove that this compactness Theorem implies the classical Selection Theorem of Mahler. We discuss generalizations of this result to ambient spaces other than $\mathbb{R}^{n}$. The space $\mathcal{U} \mathcal{D}_{r}$ is the space of equal sphere packings of radius $r / 2$. 


\section{Introduction}

In 1946 Mahler [Ma] obtained important results on star bodies and their critical lattices in $\mathbb{R}^{n}$ using the following fundamental result called now Mahler's Selection Theorem or Mahler's compactness Theorem.

Theorem 1.1. Let $\left(L_{r}\right)$ be a sequence of lattices of $\mathbb{R}^{n}$ such that, for all $r$ :

(i) $\|x\| \geq c$ for all $x \in L_{r}, x \neq 0$, with $c$ a strictly positive constant independent of $r$,

(ii) the Lebesgue measure $\left|L_{r}\right|$ of the fundamental region of $L_{r}$ satisfies $\left|L_{r}\right| \leq M$ with $M$ a constant $<+\infty$ independent of $r$.

Then one can extract from the sequence $\left(L_{r}\right)$ a subsequence $\left(L_{r^{\prime}}\right)$ that converges to a lattice $L$ of $\mathbb{R}^{n}$ such that $|L|=\lim _{r^{\prime} \rightarrow+\infty}\left|L_{r^{\prime}}\right|$.

This Theorem is very efficient in many problems of Geometry of Numbers [Ca], [GL] and is as important as the Ascoli-Arzela Theorem in Analysis. The desirability of extending the main Theorems of Geometry of numbers, whose Mahler's compactness Theorem, to general algebraic number fields and more was emphasized by Mahler in a seminar at Princeton [RSD]. Several authors revisited this Theorem, giving generalizations and analogs for other ambient spaces than $\mathbb{R}^{n}$ : Chabauty [Ch] with subgroups in locally compact abelian groups, Mumford [Mu] in semi-simple Lie groups without compact factors and moduli spaces of compact Riemann surfaces of given genus, Macbeath and Swierczkowski [MS] in locally compact and $\sigma$-compact topological groups (abelian or not) which are compactly generated, McFeat [Mf] in adele spaces of number fields, Rogers and Swinnerton-Dyer [RSD] in algebraic number fields. Groemer [Groe] gave an elegant proof of this Theorem by showing that it is a consequence of the Selection Theorem of Blaschke [Ca], by noticing the bicontinuous one-to-one correspondence between lattices and their Voronoi domains.

The way that Chabauty [Ch] proved Theorem 1.1 is extremely instructive. A careful attention to his "elementary" proof reveals the very important following fact that the $\mathbb{Z}$-additive structure of the lattices $L_{r}$ is not necessary to obtain the convergence of a subsequence. From this essential remark, Chabauty proposed in [Ch] a possible extension of Mahler's compactness Theorem to locally compact abelian groups as ambient spaces with a suitable topology, method which was improved by Mumford [Mu]. Furthermore it opens the way to deal with sequences of non-periodic point sets, that is without any additional algebraic structure, instead of only lattices or subgroups, suggesting that Mahler's Selection Theorem should exist in more general situations.

In the present note we develop a new version of Theorem 1.1 adapted to point sets (i.e. not only lattice or subgroup point sets) in an "ambient space". This can be formulated as follows. We will be interested in sets of 
point sets, say $\mathcal{U D}(H, \delta)_{r}$, of a metric space $(H, \delta)$, which is the "ambient space", where $\delta$ is a metric on $H$, which have the property that the minimal interpoint distance is greater than or equal to a given strictly positive constant, say $r$. Point sets of $H$ having this property are said uniformly discrete sets of constant $r$. Denote by $\mathcal{U D}(H, \delta)_{r, f}$ the subset of $\mathcal{U D}(H, \delta)_{r}$ formed by the finite point sets. Concerning assertion (i) in Theorem 1.1, the fundamental question is now the following:

Question 1.1. For which metric spaces $(H, \delta)$ can the set $\mathcal{U D}(H, \delta)_{r}$ be endowed with a topology such that it is compact and that the Hausdorff metric $\Delta$ on $\mathcal{U D}(H, \delta)_{r, f}$ is compatible with the restriction of this topology to $\mathcal{U D}(H, \delta)_{r, f}$ and for which values of $r$ ?

In the objective of generalizing assertion (ii) of Theorem 1.1, let us recall the (Besicovitch) concept of relative denseness [MVG]: a subset $\Lambda$ of $(H, \delta)$ is said relatively dense (for $\delta$ ) in $H$ if there exists $R>0$ such that for all $z \in H$ there exists $\lambda \in \Lambda$ such that $\delta(z, \lambda) \leq R$. We will say that $\Lambda$ is relatively dense of constant $R$ if $R$ is chosen minimal for that property. Then, assuming $H$ satisfies Question 1.1 for some $r>0$, we can formulate the second question as follows:

Question 1.2. For which metric spaces $(H, \delta)$ is the subset $X(H, \delta)_{r, R}$ of $\mathcal{U D}(H, \delta)_{r}$ of the relatively dense subsets of $H$ of given constant $R>0$ compact, and for which values of $R$ ?

By definition, a subset $\Lambda$ of $(H, \delta)$ is a Delone set if there exist $r>0$ and $R>0$ such that it is uniformly discrete of constant $\geq r$ and relatively dense of constant $R>0$. In this case we say that $\Lambda$ is a Delone set of constants $(r, R)$ (see [MVG] for possible values of $R / r$ when $H=\mathbb{R}^{n}$ ). For instance, a lattice in $\left(\mathbb{R}^{n},\|\cdot\|\right)$ is already a Delone set, where $\|\cdot\|$ is the standard euclidean metric. Note that Question 1.2 makes sense for any ambient space $(H, \delta)$ for which Delone sets are infinite, as $\left(\mathbb{R}^{n},\|\cdot\|\right)$. Indeed, if $H$ is such that its Delone sets are all finite, then Question 1.2 can be answered by the classical properties of the Hausdorff metric on the space of compact subsets of $H$ (see Section 6). The main Theorem of this note is the following (proved in Section 4). It provides answers to Question 1.1 and Question 1.2 when $H=\mathbb{R}^{n}$ and $\delta=\|\cdot\|$. For short, in this case, let us denote by $\mathcal{U} \mathcal{D}_{r}$, resp. $\mathcal{U} \mathcal{D}_{r, f}$, the set $\mathcal{U D}\left(\mathbb{R}^{n}, \delta\right)_{r}$, resp. $\mathcal{U D}\left(\mathbb{R}^{n}, \delta\right)_{r, f}$, and by $X_{r, R}$ the set $X\left(\mathbb{R}^{n}, \delta\right)_{r, R}$.

Theorem 1.2. For all $r>0$, the set $\mathcal{U} \mathcal{D}_{r}$ can be endowed with a metric $d$ such that the topological space $\left(\mathcal{U D}_{r}, d\right)$ is compact and such that the Hausdorff metric on $\mathcal{U} \mathcal{D}_{r, f}$ is compatible with the restriction of the topology of $\left(\mathcal{U} \mathcal{D}_{r}, d\right)$ to $\mathcal{U} \mathcal{D}_{r, f}$. For all $R>0$, the subspace $X_{r, R}$ of $\left(\mathcal{U} \mathcal{D}_{r}, d\right)$ of the Delone sets of constants $(r, R)$ is closed. 
Note that $\mathcal{U} \mathcal{D}_{r}$ is exactly the space of (equal) sphere packings of radius $r / 2$ of $\mathbb{R}^{n}[\mathrm{Ca}]$, [Ro].

In Section 2 a construction of $d$ is given from an averaging sequence of compact sets $\left(K_{k}\right)_{k \geq 1}$ of $\mathbb{R}^{n}$ and the corresponding Hausdorff metric on $\mathcal{U D}\left(K_{k},\|\cdot\|\right)_{r, f}, k \geq 1$.

Two other constructions of equivalent metrics are given in Section 3; the first one (Subsection 3.1) is inspired by a metric put on the space of Delone sets, which is used in tiling dynamical systems arising from Delone sets (see Radin and Wolff [RW], Robinson [Ro], Solomyak [So], Gouéré [Go], Baake and Lenz $[\mathrm{BL}]$ ); this metric is here adapted to uniformly discrete sets. The second one (Subsection 3.2) is obtained by point-counting systems normalized by suitable distances: this idea was first formulated by Dworkin in $[\mathrm{Dw}]$ (for Delone sets) though given there without any proof by the author. In this last case, since its construction is far away from the Hausdorff metric, we show in final that it implies compatibility with the Hausdorff metric on $\mathcal{U} \mathcal{D}_{r, f}$ (Corollary 3.2). The construction of this last metric may seem overly complicated at first sight, but it is of crytallographic nature, with purposes in Geometry of Numbers, while the two other metrics arise from Analysis. The third metric is adapted to study local clusters of spheres in dense sphere packings, whose geometrical classification reveals to be essential, as in Hales's works on Kepler Conjecture [Ha], [La] (see Remark in §3.3). These three metrics require a base point in the ambient space $\mathbb{R}^{n}$, which will be conveniently taken common and equal to 0 . They give a way to create new metrics on $\mathcal{U} \mathcal{D}_{r}$, for instance invariant by translations and crystallographic operations adapted to study local and global properties of aperiodic sphere packings [MVG], [MVG1]. In Subsection 3.3 we show that these metrics are topologically equivalent. This topological equivalence is deeply related to the uniform continuity of the removal process of points of a $\mathcal{U} \mathcal{D}$-set at infinity (Proposition 3.10 and Proposition A.1 in the Appendix).

In Section 5 we show that Theorem 1.2 implies Mahler's Selection Theorem 1.1 and comment in Section 6 on the space $H$ to provide positive answers to Question 1.1 and Question 1.2. In particular we extend a theorem of Macbeath and Swierczkowski [MS] to the metric case (see Theorem 6.1).

The Appendix contains a proof of the uniform continuity, for the third metric, of the removal process of points of an arbitrary $\mathcal{U}_{\mathcal{D}}$-set $\Lambda$ at infinity (Proposition A.1), given in a self-contained and detailed way in Step 2. The computations in Step 1, relative to the case $\Lambda=\mathbb{Z}^{n}$, useful in Step 2, are treated in the same way as in Step 2, therefore in a detailed way, to help the reader.

In the sequel we assume $r=1$, the general case being identical, and denote $\mathcal{U} \mathcal{D}_{1}$ by $\mathcal{U D}$, resp. $X_{1, R}$ by $X_{R}$, and by $\mathcal{U} \mathcal{D}_{f}$ the space of finite 
uniformly discrete sets of $\mathbb{R}^{n}$ of constant 1 . Elements of $\mathcal{U D}$ are called $\mathcal{U D}$-sets: they are, either the subset of $\mathbb{R}^{n}$ which contains no point, i.e. the empty set, denoted by $\emptyset$, or one-point subsets $\{x\}$ of $\mathbb{R}^{n}$, with $x \in \mathbb{R}^{n}$, or discrete point subsets $\Lambda$ of $\mathbb{R}^{n}$ which contain at least two points such that $\|x-y\| \geq 1$ as soon as $x, y \in \Lambda$, with $x \neq y$. $\mathcal{U} \mathcal{D}$-sets of $\mathbb{R}^{n}$, except the empty set $\emptyset$, may have very different $\mathbb{R}$-spans, with affine dimensions ranging from 0 to $n$. We denote by $B(c, \epsilon)$ the closed ball of $\mathbb{R}^{n}$ of center $c$ and radius $\epsilon \geq 0$, by $\stackrel{\circ}{B}(c, \epsilon)$ its interior, by $\operatorname{diam}(A)$ (resp. $\mathcal{R}_{A}$ ) the diameter (resp. the circumscribed radius $:=\sup _{a \in A}\|a\|$ ) of a nonempty subset $A$ of $\mathbb{R}^{n}$, and by $\operatorname{dist}(A, B)$ the distance $\inf \{\|a-b\| \mid a \in A, b \in B\}$ between two nonempty subsets $A$ and $B$ of $\mathbb{R}^{n}$.

\section{Construction of a metric from the Hausdorff metric}

Denote by $\Delta$ the Hausdorff metric on the space of nonempty closed subsets of $\mathbb{R}^{n}$ and by the same symbol its restriction to the space of nonempty closed subsets of any nonempty compact subset of $\mathbb{R}^{n}$ :

$$
\Delta(F, G):=\inf \{\rho \geq 0 \mid F \subset G+B(0, \rho) \text { and } G \subset F+B(0, \rho)\} .
$$

Let $\left(K_{k}\right)_{k \geq 1}$ be an averaging sequence of compacts sets of $\mathbb{R}^{n}$ which contains the base point $p_{\text {base }}:=0: K_{1} \supset\left\{p_{\text {base }}=0\right\}$ and for all $k \geq 1, K_{k} \subset K_{k+1}$, with the property $\cup_{k \geq 1} K_{k}=\mathbb{R}^{n}$. For all $k \geq 1$ and all $\Lambda, \Lambda^{\prime} \in \mathcal{U D}$ which are not simultaneously empty, we put

$$
d_{k}\left(\Lambda, \Lambda^{\prime}\right):=\Delta\left(\Lambda \cap K_{k}, \Lambda^{\prime} \cap K_{k}\right) .
$$

If $\Lambda \cap K_{k}$ or $\Lambda^{\prime} \cap K_{k}$ is empty, then $d_{k}\left(\Lambda, \Lambda^{\prime}\right)$ takes the value $+\infty$. On the contrary, since we use the convention that for all $c \in \mathbb{R}^{n}$ and all $\epsilon \geq 0$ the $\mathcal{U D}$-set $\emptyset+B(c, \epsilon)$ equals the emptyset $\emptyset$, we have:

$$
d_{k}(\emptyset, \emptyset)=0 \quad \text { for all } k \geq 0 .
$$

Then we define the mapping $d$ on $\mathcal{U D} \times \mathcal{U D}$, valued in $[0,1]$, associated with $\left(K_{k}\right)_{k \geq 1}$, by

$$
d\left(\Lambda, \Lambda^{\prime}\right):=\sum_{k \geq 1} 2^{-k} \frac{d_{k}\left(\Lambda, \Lambda^{\prime}\right)}{1+d_{k}\left(\Lambda, \Lambda^{\prime}\right)} \quad \text { for all } \Lambda, \Lambda^{\prime} \in \mathcal{U D}
$$

$\left(\right.$ with $d_{k}\left(\Lambda, \Lambda^{\prime}\right) /\left(1+d_{k}\left(\Lambda, \Lambda^{\prime}\right)\right)=1$ when $\left.d_{k}\left(\Lambda, \Lambda^{\prime}\right)=+\infty\right)$.

Proposition 2.1. The mapping $d$ is a metric on $\mathcal{U D}$. The Hausdorff metric on $\mathcal{U D}_{f}$ is compatible with the restriction of the topology of $(\mathcal{U D}, d)$ to $\mathcal{U} \mathcal{D}_{f}$.

Proof. Obvious by (2.3), and by construction for the compatibility with $\Delta$. 
Remark. If $\left(K_{k}^{\prime}\right)_{k \geq 1}$ is another averaging sequence of compact sets of $\mathbb{R}^{n}$ such that $K_{1}^{\prime}$ contains the base point 0 , the metric $d^{\prime}$ associated with $\left(K_{k}^{\prime}\right)_{k \geq 1}$ is topologically equivalent to the above metric $d$ constructed from $\left(K_{k}\right)_{k \geq 1}$ : indeed, if $\left(F_{n}\right)_{n}$ is a sequence of $\mathcal{U D}$-sets which converges to a $\mathcal{U D}$-set $F$ for the metric $d^{\prime}$, i.e. $d^{\prime}\left(F_{n}, F\right) \rightarrow 0, n \rightarrow \infty$, then, for all $k \geq 1,\left(F_{n} \cap K_{k}^{\prime}\right)_{n}$ is a Cauchy sequence in $\left(\mathcal{U D}\left(K_{k}^{\prime},\|\cdot\|\right)_{1}, \Delta\right)$. If $j_{k}$ is the greatest integer $l$ such that $K_{l} \subset K_{k}^{\prime}$, then $\left(F_{n} \cap K_{j_{k}}\right)_{n}$ is a Cauchy sequence in $\left(\mathcal{U D}\left(K_{j_{k}},\|\cdot\|\right)_{1}, \Delta\right)$ which converges to $F \cap K_{j_{k}}$. Since $\cup_{k \geq 1} F \cap K_{k}^{\prime}=\cup_{k \geq 1} F \cap K_{\jmath_{k}}=\cup_{k \geq 1} F \cap K_{k}=F$, for all $k \geq 1, d_{j_{k}}\left(F_{n}, F\right)$ tends to 0 when $n$ tends to infinity. We deduce $\lim _{n \rightarrow+\infty} d\left(F_{n}, F\right)=0$ by (2.4) and Lebesgue dominated convergence theorem. Therefore, to obtain a distance $d$ with properties easy to describe, it suffices to consider an averaging sequence of balls centered at the base point 0 of $\mathbb{R}^{n}$ : for instance, $K_{k}=B\left(0, R_{k}\right), k \geq 1$, with $\left(R_{k}\right)_{k \geq 1}$ a strictly increasing sequence satisfying $\lim _{k \rightarrow+\infty} R_{k}=+\infty$.

Let us note that if $2^{-k}$ is replaced by $a_{k}$ in (2.4) where $0 \leq a_{k}$ and $\sum_{k \geq 1} a_{k}<+\infty$, we obtain another metric which is also topologically equivalent to $d$. All these possibilities constitute the class of metrics of $d$.

A discrete subset $\Lambda$ of $\mathbb{R}^{n}$ is said locally finite if $\Lambda \cap B(c, \epsilon)$ is finite for all $c \in \mathbb{R}^{n}$ and all $\epsilon>0$. The distance $d$ can be extended to the space of locally finite subsets of $\mathbb{R}^{n}$. Denote by $\mathcal{D}_{l f}\left(\mathbb{R}^{n},\|\cdot\|\right)$ this space. Note that $\emptyset \in \cup_{r>0} \mathcal{U} \mathcal{D}_{r}$ and that $\cup_{r>0} \mathcal{U} \mathcal{D}_{r}$ is contained in $\mathcal{D}_{l f}\left(\mathbb{R}^{n},\|\cdot\|\right)$.

Proposition 2.2. The mapping $d$ associated with an averaging sequence of compact sets $\left(K_{k}\right)_{k \geq 1}$ of $\mathbb{R}^{n}$ is a metric on the space $\mathcal{D}_{l f}\left(\mathbb{R}^{n},\|\cdot\|\right)$ of locally finite discrete subsets of $\mathbb{R}^{n}$. The Hausdorff metric on $\cup_{r>0} \mathcal{U} \mathcal{D}_{r, f}$ is compatible with the restriction of the topology of $\left(\mathcal{D}_{l f}\left(\mathbb{R}^{n},\|\cdot\|\right), d\right)$ to $\cup_{r>0} \mathcal{U} \mathcal{D}_{r, f}$.

Proof. Same construction and arguments as in Proposition 2.1.

\section{Equivalent metrics}

3.1. From tiling dynamical systems. Let $R_{\min }>0$ be defined by the following property: $X_{R}=\emptyset$ if $R<R_{\min }$. It is the smallest possible Delone constant of any Delone set (with minimal interpoint distance $\geq 1$ ) in $\mathbb{R}^{n}$, and depends only upon $n$ [MVG]. It is linked to packings of equal spheres of radius $1 / 2$ in $\mathbb{R}^{n}$ exhibiting spherical holes whose radius is always smaller than or equal to $R_{\min }$ and therefore to densest sphere packings [MVG], [CS]. Let $\lambda \geq 2 R_{\min }^{2}$ and $p_{\text {base }}=0$ the base point of $\mathbb{R}^{n}$. Then, for all $\Lambda, \Lambda^{\prime} \in \mathcal{U D}$ denote: 


$$
\begin{aligned}
\Omega\left(\Lambda, \Lambda^{\prime}\right):=\left\{\alpha>0 \mid \Lambda \cap B\left(p_{\text {base }}, \frac{\lambda}{\alpha}\right) \subset \Lambda^{\prime}+B(0, \alpha)\right. \\
\text { and } \left.\Lambda^{\prime} \cap B\left(p_{\text {base }}, \frac{\lambda}{\alpha}\right) \subset \Lambda+B(0, \alpha)\right\}
\end{aligned}
$$

and define

$$
\delta_{1}\left(\Lambda, \Lambda^{\prime}\right):=\min \left\{1, \frac{\inf \Omega\left(\Lambda, \Lambda^{\prime}\right)}{R_{\min }}\right\} .
$$

Observe that, if $\alpha \in \Omega\left(\Lambda, \Lambda^{\prime}\right)$, then $[\alpha,+\infty) \subset \Omega\left(\Lambda, \Lambda^{\prime}\right)$. We have: $\delta_{1}(\emptyset, \emptyset)=0$ and, for all $\Lambda \neq \emptyset, \delta_{1}(\Lambda, \emptyset)=\min \left\{1, \frac{\lambda}{R_{\min } \operatorname{dist}(\{0\}, \Lambda)}\right\}$.

Proposition 3.1. The mapping $\delta_{1}$ is a metric on $\mathcal{U D}$. The Hausdorff metric on $\mathcal{U D}_{f}$ is compatible with the restriction of the topology of $\left(\mathcal{U D}, \delta_{1}\right)$ to $\mathcal{U} \mathcal{D}_{f}$.

Proof. It is obviously symmetrical. If $\Lambda=\Lambda^{\prime}$, then $\delta_{1}\left(\Lambda, \Lambda^{\prime}\right)=0$. Let us show the converse. Assume $\delta_{1}\left(\Lambda, \Lambda^{\prime}\right)=0$. If $\Lambda$ or $\Lambda^{\prime}$ is the empty set, then it is easy to show that both are equal to $\emptyset$. Assume now that $\Lambda \neq \emptyset, \Lambda^{\prime} \neq \emptyset$ and that $\Lambda$ strictly contains $\Lambda \cap \Lambda^{\prime}$. Then there exists $x \in \Lambda, x \notin \Lambda^{\prime}$ such that $\operatorname{dist}\left(\{x\}, \Lambda^{\prime}\right)>0$. Since $\Omega\left(\Lambda, \Lambda^{\prime}\right)$ equals $(0,+\infty)$ by assumption, it contains in particular $\frac{1}{2} \operatorname{dist}(\{x\}, \Lambda)>0$ and also $\lambda /(2\|x\|)>0$ if $x \neq 0$. Take $\beta:=\frac{1}{2} \min \left\{\operatorname{dist}\left(\{x\}, \Lambda^{\prime}\right), \lambda /\|x\|\right\}$ when $x \neq 0$, and $\beta:=\frac{1}{2} \operatorname{dist}\left(\{0\}, \Lambda^{\prime}\right)$ when $x=0$. Then we would have: $x \in \Lambda \cap B\left(0, \frac{\lambda}{\beta}\right)$ but $x \notin \Lambda^{\prime}+B(0, \beta)$. Contradiction. Therefore, $\Lambda=\Lambda \cap \Lambda^{\prime}$, equivalently $\Lambda \subset \Lambda^{\prime}$. In a similar way, by symmetry, we obtain $\Lambda^{\prime} \subset \Lambda$, hence the equality $\Lambda=\Lambda^{\prime}$.

Let us show the triangle inequality:

$$
\delta_{1}\left(\Lambda, \Lambda^{\prime \prime}\right) \leq \delta_{1}\left(\Lambda, \Lambda^{\prime}\right)+\delta_{1}\left(\Lambda^{\prime}, \Lambda^{\prime \prime}\right) .
$$

If $\delta_{1}\left(\Lambda, \Lambda^{\prime}\right)=1$ or if $\delta_{1}\left(\Lambda^{\prime}, \Lambda^{\prime \prime}\right)=1$, then it is satisfied. Assume now $\delta_{1}\left(\Lambda, \Lambda^{\prime}\right)<1$ and $\delta_{1}\left(\Lambda^{\prime}, \Lambda^{\prime \prime}\right)<1$. Let $a \in \Omega\left(\Lambda, \Lambda^{\prime}\right)$ and $b \in \Omega\left(\Lambda^{\prime}, \Lambda^{\prime \prime}\right)$. Then $a<R_{\text {min }}$ and $b<R_{\text {min }}$. Let $e=a+b$. Then

$$
\Lambda \cap B\left(0, \frac{\lambda}{e}\right) \subset \Lambda \cap B\left(0, \frac{\lambda}{a}\right) \subset \Lambda^{\prime}+B(0, a) .
$$

This implies:

$$
\Lambda \cap B\left(0, \frac{\lambda}{e}\right) \subset \Lambda^{\prime} \cap B\left(0, \frac{\lambda}{e}+a\right)+B(0, a) .
$$

But $a+\frac{\lambda}{e} \leq \frac{\lambda}{b}$ : indeed, since $b e \leq 2 R_{\min }^{2}$, we have: $\frac{\lambda}{b}-\frac{\lambda}{e}-a=\frac{a}{b e}(\lambda-b e) \geq 0$. Hence, $\Lambda \cap B\left(0, \frac{\lambda}{e}\right) \subset \Lambda^{\prime} \cap B\left(0, \frac{\lambda}{b}\right)+B(0, a) \subset \Lambda^{\prime \prime}+B(0, b)+B(0, a)=\Lambda^{\prime \prime}+B(0, e)$. 
Therefore $e \in \Omega\left(\Lambda, \Lambda^{\prime \prime}\right)$, that is $\Omega\left(\Lambda, \Lambda^{\prime \prime}\right) \supset \Omega\left(\Lambda, \Lambda^{\prime}\right)+\Omega\left(\Lambda^{\prime}, \Lambda^{\prime \prime}\right)$. This implies the triangle inequality.

To prove the compatibility of the Hausdorff metric $\Delta$ on $\mathcal{U} \mathcal{D}_{f}$ with the topology arising from $\delta_{1}$, it suffices to show, given $\Lambda, \Lambda^{\prime} \in \mathcal{U} \mathcal{D}_{f}$ such that $\delta_{1}\left(\Lambda, \Lambda^{\prime}\right)$ is small enough, that the following equality holds:

$$
\delta_{1}\left(\Lambda, \Lambda^{\prime}\right)=\frac{\Delta\left(\Lambda, \Lambda^{\prime}\right)}{R_{\min }} .
$$

Indeed, if $\delta_{1}\left(\Lambda, \Lambda^{\prime}\right)$ is small enough, then there exists $T \in \Omega\left(\Lambda, \Lambda^{\prime}\right)$ such that $\Lambda=\Lambda \cap B\left(0, \frac{\lambda}{T}\right)$ and $\Lambda^{\prime}=\Lambda^{\prime} \cap B\left(0, \frac{\lambda}{T}\right)$. Thus

$$
\inf \Omega\left(\Lambda, \Lambda^{\prime}\right)=\inf \left\{\rho \geq 0 \mid \Lambda \subset \Lambda^{\prime}+B(0, \rho)\right.
$$

and

$$
\left.\Lambda^{\prime} \subset \Lambda+B(0, \rho)\right\}=\Delta\left(\Lambda, \Lambda^{\prime}\right)
$$

We deduce (3.3).

Proposition 3.2. The mapping $\delta_{1}$ is a metric on the space $\mathcal{D}_{l f}\left(\mathbb{R}^{n},\|\cdot\|\right)$ of locally finite discrete subsets of $\mathbb{R}^{n}$. The Hausdorff metric on $\cup_{r>0} \mathcal{U} \mathcal{D}_{r, f}$ is compatible with the restriction of the topology of $\left(\mathcal{D}_{l f}\left(\mathbb{R}^{n},\|\cdot\|\right), \delta_{1}\right)$ to $\cup_{r>0} \mathcal{U} \mathcal{D}_{r, f}$.

Proof. Same construction as in Proposition 3.1.

Remark. After Blichfeldt (see [MVG]) we have: $R_{\min } \geq \frac{\sqrt{2}}{2} \sqrt{\frac{n}{n+1}}$, hence $\lambda \geq \frac{n}{n+1}$. Given $\Lambda \in X_{R}$, with $R \geq R_{\min }$, denote by $\Lambda^{(d h)}$ the uniformly discrete set of $\mathbb{R}^{n}$ constituted by the deepest holes of $\Lambda$ [CS]. Consider the class of metrics $\delta_{1}$ constructed as above when $\lambda \geq 2 R_{\min }^{2}$ varies. The normalization factor $R_{\min }^{-1}$ in the definition of $\delta_{1}$ comes from the fact that, for all $\Lambda \in X_{R}$ with $R \geq R_{\min }$, we have: $\delta_{1}\left(\Lambda, \Lambda^{(d h)}\right)=1$ for all $\lambda$ large enough.

3.2. From point-counting systems with equal spheres. Contrarily to $d$ and $\delta_{1}$ the metric $\delta_{2}$ constructed here on $\mathcal{U D}$ has no natural extension to $\mathcal{D}_{l f}\left(\mathbb{R}^{n},\|\cdot\|\right)$. But it possesses nice properties (Subsection 3.2.2) like the point pairing property (Proposition 3.6).

3.2.1. Construction. Let $\mathcal{E}=\left\{(D, E) \mid D\right.$ countable point set in $\mathbb{R}^{n}, E$ countable point set in $\left.\left(0, \frac{1}{2}\right)\right\}$ and $f: \mathbb{R}^{n} \rightarrow[0,1]$ a continuous function with compact support in $B(0,1)$ which satisfies $f(0)=1$ and $f(t) \leq \frac{1 / 2+\|\lambda-t / 2\|}{1 / 2+\|\lambda\|}$ for all $t \in B(0,1)$ and $\lambda \in \mathbb{R}^{n}$ (for technical reasons which will appear below; it is a weak hypothesis; take for instance $f(t)=1-\|t\|$ on $B(0,1)$ and $f(t)=0$ elsewhere). Recall that a pseudo-metric $\delta$ on a space satisfies 
all the axioms of a distance except that $\delta(u, v)=0$ does not necessarily imply $u=v$.

With each element $(D, E) \in \mathcal{E}$ and (variable) origin $\alpha$ of the affine euclidean space $\mathbb{R}^{n}$ we associate a real-valued function $d_{\alpha,(D, E)}$ on $\mathcal{U D} \times \mathcal{U D}$ in the following way. Let $\mathcal{B}_{(D, E)}=\left\{\mathcal{B}_{m}\right\}$ denote the countable set of all possible finite collections $\mathcal{B}_{m}=\left\{\stackrel{\circ}{B}\left(c_{1}^{(m)}, \epsilon_{1}^{(m)}\right), \stackrel{\circ}{B}\left(c_{2}^{(m)}, \epsilon_{2}^{(m)}\right), \ldots, \stackrel{\circ}{B}\left(c_{i_{m}}^{(m)}, \epsilon_{i_{m}}^{(m)}\right)\right\}$ (with $i_{m}$ the number of elements $\# \mathcal{B}_{m}$ of $\mathcal{B}_{m}$ ) of open balls such that $c_{q}^{(m)} \in D$ and $\epsilon_{q}^{(m)} \in E$ for all $q \in\left\{1,2, \ldots, i_{m}\right\}$, and such that for all $m$ and any pair of balls in $\mathcal{B}_{m}$ of respective centers $c_{q}^{(m)}$ and $c_{k}^{(m)}$, we have $\left\|c_{q}^{(m)}-c_{k}^{(m)}\right\| \geq 1$. Since any $\mathcal{U D}$-set $\Lambda$ is countable, we denote by $\Lambda_{i}$ its $i$-th element. Then we define the following function, with $\Lambda, \Lambda^{\prime} \in \mathcal{U D}$, and base point $p_{\text {base }}=0$ :

$$
\begin{aligned}
& d_{\alpha,(D, E)}\left(\Lambda, \Lambda^{\prime}\right) \\
& :=\sup _{\mathcal{B}_{m} \in \mathcal{B}_{(D, E)}} \frac{\left|\phi_{\mathcal{B}_{m}}(\Lambda)-\phi_{\mathcal{B}_{m}}\left(\Lambda^{\prime}\right)\right|}{\left(1 / 2+\left\|\alpha-p_{\text {base }}\right\|+\left\|\alpha-c_{1}^{(m)}\right\|+\left\|\alpha-c_{2}^{(m)}\right\|+\cdots+\left\|\alpha-c_{i_{m}}^{(m)}\right\|\right)}
\end{aligned}
$$

where the real-valued function $\phi_{\mathcal{B}_{m}}$ is given by

$$
\phi_{\mathcal{B}_{m}}(\Lambda):=\sum_{\substack{\circ \\ B(c, \epsilon) \in \mathcal{B}_{m}}} \sum_{i} \epsilon f\left(\frac{\Lambda_{i}-c}{\epsilon}\right) .
$$

By convention we put $\phi_{\mathcal{B}_{m}}(\emptyset)=0$ for all $\mathcal{B}_{m} \in \mathcal{B}_{(D, E)}$ and all $(D, E) \in \mathcal{E}$. It is clear that, for all $m$ and $\Lambda \in \mathcal{U D}$, inside each ball $\stackrel{\circ}{B}(c, \epsilon) \in \mathcal{B}_{m}$, there is at most one point of $\Lambda$ and therefore the summation $\sum_{i} \epsilon f\left(\frac{\Lambda_{i}-c}{\epsilon}\right)$ is reduced to at most one non-zero term. Therefore the sum $\phi_{\mathcal{B}_{m}}(\Lambda)$ is finite.

Lemma 3.1. For all $(\alpha,(D, E))$ in $\mathbb{R}^{n} \times \mathcal{E}, d_{\alpha,(D, E)}$ is a pseudo-metric valued in $[0,1]$.

Proof. Let $\alpha \in \mathbb{R}^{n}$ and $(D, E) \in \mathcal{E}$. It is easy to check that $d_{\alpha,(D, E)}$ is a pseudo-metric on $\mathcal{U D}$. Let us show it is valued in $[0,1]$. Let us consider $\mathcal{B}_{m} \in \mathcal{B}_{(D, E)}$ for which the centers of its constitutive balls are denoted by $c_{1}, c_{2}, \ldots, c_{i_{m}}$. Then we have

$$
\frac{i_{m}}{2} \leq 1 / 2+\|\alpha\|+\left\|\alpha-c_{1}\right\|+\left\|\alpha-c_{2}\right\|+\cdots+\left\|\alpha-c_{i_{m}}\right\| .
$$

Indeed, if there exists $j \in\left\{1,2, \ldots, i_{m}\right\}$ such that $\left\|c_{j}-\alpha\right\| \leq \frac{1}{2}$, then for all $k \neq j,\left\|c_{k}-\alpha\right\| \geq \frac{1}{2}$. Hence

$\frac{1}{2}+\|\alpha\|+\left\|\alpha-c_{1}\right\|+\left\|\alpha-c_{2}\right\|+\cdots+\left\|\alpha-c_{i_{m}}\right\| \geq \frac{1}{2}+\|\alpha\|+\frac{i_{m}-1}{2} \geq \frac{i_{m}}{2}$. 
If $\left\|c_{k}-\alpha\right\| \geq \frac{1}{2}$ for all $k \in\left\{1,2, \ldots, i_{m}\right\}$, then

$$
\frac{1}{2}+\|\alpha\|+\left\|\alpha-c_{1}\right\|+\left\|\alpha-c_{2}\right\|+\cdots+\left\|\alpha-c_{i_{m}}\right\| \geq \frac{1}{2}+\|\alpha\|+\frac{i_{m}}{2} \geq \frac{i_{m}}{2} .
$$

On the other hand, since the radii of the balls $\stackrel{\circ}{B}\left(c_{j}, \epsilon_{j}\right) \in \mathcal{B}_{m}$ are less than $\frac{1}{2}$ by construction, we have $0 \leq \phi_{\mathcal{B}_{m}}(\Lambda) \leq \frac{i_{m}}{2}$ for all $\mathcal{U} \mathcal{D}$-set $\Lambda$. Therefore $\left|\phi_{\mathcal{B}_{m}}(\Lambda)-\phi_{\mathcal{B}_{m}}\left(\Lambda^{\prime}\right)\right| \leq \frac{1}{2}+\|\alpha\|+\left\|\alpha-c_{1}\right\|+\left\|\alpha-c_{2}\right\|+\cdots+\left\|\alpha-c_{i_{m}}\right\|$, for all $\mathcal{B}_{m} \in \mathcal{B}_{(D, E)}$ and all $\mathcal{U} \mathcal{D}$-sets $\Lambda, \Lambda^{\prime}$. We deduce the claim.

The uniform topology on $\mathcal{U D}$ given by the pseudo-metrics $d_{\alpha,(D, E)}$ is generated by the open sets $\left\{\Lambda \in \mathcal{U D} \mid d_{\alpha,(D, E)}(u, \Lambda)<\epsilon\right\}, u \in \mathcal{U D}$ (Weil [We]). In order to get rid of a peculiar choice of the (variable) origin $\alpha$ and of the element $(D, E)$ of $\mathcal{E}$, the supremum over all choices $(\alpha,(D, E))$ in $\mathbb{R}^{n} \times \mathcal{E}$ is taken.

Proposition 3.3. The supremum $\delta_{2}:=\sup _{\alpha \in \mathbb{R}^{n},(D, E) \in \mathcal{E}} d_{\alpha,(D, E)}$ is a metric on $\mathcal{U D}$, valued in $[0,1]$.

Proof. The supremum of the family of pseudo-metrics $d_{\alpha,(D, E)}$ is a pseudometric which takes its values in $[0,1]$. We have to show that $\delta_{2}$ is a metric. Assume $\Lambda, \Lambda^{\prime}$ are $\mathcal{U D}$-sets which are not empty such that $\delta_{2}\left(\Lambda, \Lambda^{\prime}\right)=0$ and let us show that $\Lambda=\Lambda^{\prime}$. We will show that $\Lambda \not \subset \Lambda^{\prime}$ and $\Lambda^{\prime} \not \subset \Lambda$ are impossible. Assume that $\Lambda \neq \Lambda^{\prime}$ and that $\Lambda \not \subset \Lambda^{\prime}$. Then there exists $\lambda \in \Lambda$ such that $\lambda \notin \Lambda^{\prime}$. Denote $\epsilon:=\frac{1}{2} \min \left\{\frac{1}{2}, \min \left\{\|\lambda-u\| \mid u \in \Lambda^{\prime}\right\}\right\}$.

We have $\epsilon>0$ since $\Lambda^{\prime}$ is a $\mathcal{U} \mathcal{D}$-set. The ball $\stackrel{\circ}{B}(\lambda, \epsilon)$ contains no point of $\Lambda^{\prime}$ and only the point $\lambda$ of $\Lambda$. Take $\alpha=\lambda, D=\{\lambda\}, E=\{\epsilon\}$. We have $d_{\lambda,(D, E)}\left(\Lambda, \Lambda^{\prime}\right)=\frac{\epsilon}{1 / 2+\|\lambda\|}>0$. Hence $\delta_{2}\left(\Lambda, \Lambda^{\prime}\right)$ would be strictly positive. Contradiction. Therefore $\Lambda \subset \Lambda^{\prime}$. Then, exchanging $\Lambda$ and $\Lambda^{\prime}$, we have $\Lambda^{\prime} \subset \Lambda$. We deduce the equality $\Lambda=\Lambda^{\prime}$. If we assume that one of the $\mathcal{U D}$-sets $\Lambda$ or $\Lambda^{\prime}$ is the empty set, we see that the above proof is still valid.

\subsubsection{Properties.}

Proposition 3.4. For all $A, B, C \in \mathcal{U D}$ such that $A \cup B \in \mathcal{U D}$ and all $(D, E) \in \mathcal{E}$ and $\mathcal{B}_{m} \in \mathcal{B}_{(D, E)}$, the following assertions hold:

(i) $\phi_{\mathcal{B}_{m}}(A \cup B)+\phi_{\mathcal{B}_{m}}(A \cap B)=\phi_{\mathcal{B}_{m}}(A)+\phi_{\mathcal{B}_{m}}(B)$;

(ii) $\delta_{2}(A \cup B, C) \leq \delta_{2}(A, C)+\delta_{2}(B, A \cap B)$;

(iii) $\delta_{2}(A \cap B, C) \leq \delta_{2}(A, C)+\delta_{2}(B, A \cup B)$.

In particular:

(iv) $\delta_{2}(A \cup B, \emptyset) \leq \delta_{2}(A, \emptyset)+\delta_{2}(B, \emptyset)$ as soon as $A \cap B=\emptyset$; 
(v) $\delta_{2}(A \cup B, A \cap B) \leq \min \left\{\delta_{2}(A, A \cap B)+\delta_{2}(B, A \cap B), \delta_{2}(A, A \cup B)+\right.$ $\left.\delta_{2}(B, A \cup B)\right\}$

(vi) if $B$ is reduced to one point, say $\{\lambda\}$, such that $\lambda \notin A$, we have: $\delta_{2}(A \cup\{\lambda\}, C) \leq \min \left\{\delta_{2}(A, C)+\delta_{2}(\{\lambda\}, \emptyset), \delta_{2}(\{\lambda\}, C)+\delta_{2}(A, \emptyset)\right\}$.

Proof. Assertion (i) can easily be checked from the definition of $\phi_{\mathcal{B}_{m}}$. Assertion (ii) is a consequence of (i) and of the inequality

$$
\begin{aligned}
\left|\phi_{\mathcal{B}_{m}}(A \cup B)-\phi_{\mathcal{B}_{m}}(C)\right| & =\left|\phi_{\mathcal{B}_{m}}(A)+\phi_{\mathcal{B}_{m}}(B)-\phi_{\mathcal{B}_{m}}(A \cap B)-\phi_{\mathcal{B}_{m}}(C)\right| \\
& \leq\left|\phi_{\mathcal{B}_{m}}(A)-\phi_{\mathcal{B}_{m}}(C)\right|+\left|\phi_{\mathcal{B}_{m}}(B)-\phi_{\mathcal{B}_{m}}(A \cap B)\right| .
\end{aligned}
$$

Assertion (iii) follows from (ii) by exchanging " $\cup$ " and " $\cap$ ". Assertions (iv) to (vi) can be deduced from (i), (ii) and (iii).

Assertions (iv) and (vi) in Proposition 3.4 show the special role played by the "empty set" element $\emptyset$ in the set-theoretic processes of "point addition" and "point removal". The uniform continuity of the "point removal process" at infinity of the points of a $\mathcal{U D}$-set is proved in Section 3.3 and in the Appendix.

Proposition 3.5. The following equalities hold:

(i) $\delta_{2}(\{\lambda\}, \emptyset)=\frac{1}{1+2\|\lambda\|}$, for all $\lambda \in \mathbb{R}^{n}$ (remarkably this value does not depend upon $f(x))$,

(ii) $\delta_{2}(\Lambda-\{\lambda\}, \Lambda)=\frac{1}{1+2\|\lambda\|}$ for all non-empty $\mathcal{U D}$-set $\Lambda$ and all $\lambda \in \Lambda$.

Proof. (i) First, let us show that $\delta_{2}(\{\lambda\}, \emptyset) \leq \frac{1}{1+2\|\lambda\|}$. By definition we have

$$
=\sup _{\substack{\alpha \in \mathbb{R}^{n} \\(D, E) \in \mathcal{E}}} \sup _{\mathcal{B}_{m} \in \mathcal{B}_{(D, E)}} \frac{\phi_{\mathcal{B}_{m}}(\Lambda)}{\left(\frac{1}{2}+\|\alpha\|+\left\|\alpha-c_{1}^{(m)}\right\|+\left\|\alpha-c_{2}^{(m)}\right\|+\cdots+\left\|\alpha-c_{j_{m}}^{(m)}\right\|\right)} .
$$

Whatever $(D, E) \in \mathcal{E}, \mathcal{B}_{m} \in \mathcal{B}_{(D, E)}$, a maximum of one ball of $\mathcal{B}_{m}$ may contain $\lambda$. Denote by $\stackrel{\circ}{B}(c, \epsilon)$ this variable generic ball and put $c=c_{1}^{(m)}$. The other balls of $\mathcal{B}_{m}$ have a zero contribution to the numerator $\phi_{\mathcal{B}_{m}}(\Lambda)$ in the expression of $\delta_{2}(\{\lambda\}, \emptyset)$. The denominator is such that: $\frac{1}{2}+\|\alpha\|+$ $\left\|\alpha-c_{1}^{(m)}\right\|+\left\|\alpha-c_{2}^{(m)}\right\|+\cdots+\left\|\alpha-c_{j_{m}}^{(m)}\right\| \geq \frac{1}{2}+\|\alpha\|+\|\alpha-c\|$. But $\frac{1}{2}+\|\alpha\|+\|\alpha-c\| \geq \frac{1}{2}+\|c\|$, this minimum being reached on the segment $[0, c]$. Therefore, by definition of the function $f$, we have

$$
d_{\alpha,(D, E)}(\{\lambda\}, \emptyset) \leq \frac{\epsilon f\left(\frac{\lambda-c}{\epsilon}\right)}{\frac{1}{2}+\|c\|} \leq \frac{\epsilon}{\frac{1}{2}+\|\lambda\|} \leq \frac{1 / 2}{1 / 2+\|\lambda\|}=\frac{1}{1+2\|\lambda\|} .
$$

Conversely, if we take $\alpha=\lambda, D=\{\lambda\}$ and $E$ a dense subset in $\left(0, \frac{1}{2}\right)$, we see that $\delta_{2}(\{\lambda\}, \emptyset) \geq d_{\alpha=\lambda,(D=\{\lambda\}, E)}(\{\lambda\}, \emptyset)=\frac{1 / 2}{1 / 2+\|\lambda\|}$. We deduce the equality and assertion (i); 
(ii) The proof is similar as in (i) since $\Lambda$ and $\Lambda-\{\lambda\}$ differ by only one element $\lambda$ which belongs to at most one ball in a collection $\mathcal{B}_{m}$ for any $(D, E) \in \mathcal{E}$ and any $\mathcal{B}_{m} \in \mathcal{B}_{(D, E)}$. The details are left to the reader.

Corollary 3.1. For all $\mathcal{U D}$-set $\Lambda \neq \emptyset$ and all $\lambda \in \Lambda$, the inequality holds:

$$
\left|\delta_{2}(\Lambda, \emptyset)-\delta_{2}(\Lambda-\{\lambda\}, \emptyset)\right| \leq \frac{1}{1+2\|\lambda\|} .
$$

Proof. From Proposition 3.4 (ii), we deduce $\delta_{2}(\Lambda, \emptyset) \leq \delta_{2}(\Lambda-\{\lambda\}, \emptyset)+$ $\delta_{2}(\{\lambda\}, \emptyset)$. From (iii) in Proposition 3.4, we obtain

$$
\delta_{2}(\Lambda-\{\lambda\}, \emptyset) \leq \delta_{2}(\Lambda, \emptyset)+\delta_{2}(\Lambda-\{\lambda\}, \Lambda)
$$

but $\delta_{2}(\{\lambda\}, \emptyset)=\delta_{2}(\Lambda-\{\lambda\}, \Lambda)=\frac{1}{1+2\|\lambda\|}$ by Proposition 3.5. We deduce the claim.

Proposition 3.6 (Point pairing property). Let $\Lambda, \Lambda^{\prime}$ be two $\mathcal{U D}$-sets assumed to be nonempty. Let $l=\operatorname{dist}(\{0\}, \Lambda)<+\infty$ and $\epsilon \in\left(0, \frac{1}{1+2 l}\right)$. Assume that $\delta_{2}\left(\Lambda, \Lambda^{\prime}\right)<\epsilon$. Then, for all $\lambda \in \Lambda$ such that $\|\lambda\|<\frac{1-\epsilon}{2 \epsilon}$,

(i) there exists a unique $\lambda^{\prime} \in \Lambda^{\prime}$ such that $\left\|\lambda^{\prime}-\lambda\right\|<\frac{1}{2}$,

(ii) this pairing $\left(\lambda, \lambda^{\prime}\right)$ satisfies the inequality: $\left\|\lambda^{\prime}-\lambda\right\| \leq\left(\frac{1}{2}+\|\lambda\|\right) \epsilon$.

Proof. (i) Let us assume that for all $\lambda^{\prime} \in \Lambda^{\prime}, \lambda \in \Lambda$, such that $\|\lambda\|<\frac{1-\epsilon}{2 \epsilon}$ the inequality $\left\|\lambda^{\prime}-\lambda\right\| \geq \frac{1}{2}$ holds. This will lead to a contradiction. Assume the existence of an element $\lambda \in \Lambda$ such that $\|\lambda\|<\frac{1-\epsilon}{2 \epsilon}$ and take $D=\{\lambda\}$ and let $E$ be a countable dense subset in $\left(0, \frac{1}{2}\right)$. Each $\mathcal{B}_{m}$ in $\mathcal{B}_{(D, E)}$ is a set constituted by only one element: the ball (say) $\stackrel{\circ}{B}\left(\lambda, e_{m}\right)$ with $e_{m} \in E$. We deduce that $\phi_{\mathcal{B}_{m}}(\Lambda)=e_{m}$ and $\phi_{\mathcal{B}_{m}}\left(\Lambda^{\prime}\right)=0$. Hence

$$
d_{\lambda,(D, E)}\left(\Lambda, \Lambda^{\prime}\right)=\sup _{m} \frac{e_{m}}{1 / 2+\|\lambda\|}=\frac{1 / 2}{1 / 2+\|\lambda\|} \leq \delta_{2}\left(\Lambda, \Lambda^{\prime}\right) .
$$

But $\epsilon<\frac{1}{1+2\|\lambda\|}$ is equivalent to $\|\lambda\|<\frac{1-\epsilon}{2 \epsilon}$. Since we have assumed $\delta_{2}\left(\Lambda, \Lambda^{\prime}\right)<\epsilon$, we should obtain $\epsilon<d_{\lambda,(D, E)}\left(\Lambda, \Lambda^{\prime}\right) \leq \delta_{2}\left(\Lambda, \Lambda^{\prime}\right)<\epsilon$. Contradiction. The uniqueness of $\lambda^{\prime}$ comes from the fact that $\Lambda^{\prime}$ is a $\mathcal{U D}$-set allowing only one element $\lambda^{\prime}$ close to $\lambda$. (ii) Let us assume that $\lambda \neq \lambda^{\prime}$ for all $\lambda \in \Lambda$ such that $\|\lambda\|<\frac{1-\epsilon}{2 \epsilon}$, with $\lambda^{\prime} \in \Lambda^{\prime}$ that satisfies $\left\|\lambda^{\prime}-\lambda\right\|<\frac{1}{2}$ (if the equality $\lambda=\lambda^{\prime}$ holds, there is nothing to prove). Then, for all $\lambda \in \Lambda$ such that $\|\lambda\|<\frac{1-\epsilon}{2 \epsilon}$, let us take $\alpha=\lambda$ as base point, $D=\{\lambda\}$ and $E$ a dense subset in $\left(0,\left\|\lambda-\lambda^{\prime}\right\|\right] \subset\left(0, \frac{1}{2}\right)$. Then $\phi_{\mathcal{B}_{m}}(\Lambda)-\phi_{\mathcal{B}_{m}}\left(\Lambda^{\prime}\right)=e_{m}\left(1-f\left(\frac{\lambda^{\prime}-\lambda}{e_{m}}\right)\right)$. The restriction of the function $z \rightarrow z\left(1-f\left(\frac{\lambda^{\prime}-\lambda}{z}\right)\right)$ to $\left(0,\left\|\lambda-\lambda^{\prime}\right\|\right]$ is the identity function and is bounded above by $\left\|\lambda^{\prime}-\lambda\right\|$. Therefore,

$$
d_{\lambda,(D, E)}\left(\Lambda, \Lambda^{\prime}\right)=\sup _{\mathcal{B}_{m}} \frac{\left|\phi_{\mathcal{B}_{m}}(\Lambda)-\phi_{\mathcal{B}_{m}}\left(\Lambda^{\prime}\right)\right|}{1 / 2+\|\lambda\|}=\frac{\left\|\lambda^{\prime}-\lambda\right\|}{1 / 2+\|\lambda\|} .
$$


Since $d_{\lambda,(D, E)}\left(\Lambda, \Lambda^{\prime}\right) \leq \delta_{2}\left(\Lambda, \Lambda^{\prime}\right)<\epsilon$, we obtain $\left\|\lambda^{\prime}-\lambda\right\| \leq\left(\frac{1}{2}+\|\lambda\|\right) \epsilon$.

In other terms, each time two $\mathcal{U D}$-sets $\Lambda, \Lambda^{\prime}$ are sufficiently close to each other for the metric $\delta_{2}$, every element of $\Lambda$ lying in a large ball centered at the origin (base point) in $\mathbb{R}^{n}$, is automatically associated with a unique element of $\Lambda^{\prime}$ which is close to it within distance less than $\frac{1}{2}$. Such pairings of elements occur over larger and larger distances from the origin when $\Lambda^{\prime}$ tends to $\Lambda$. From (ii) the proximity in the pairings $\left(\lambda, \lambda^{\prime}\right)$ is much better for the elements $\lambda \in \Lambda$ which are the closest to the base point.

Proposition 3.7. Let $\epsilon \in(0,1)$ and $\Lambda \in \mathcal{U} \mathcal{D}, \Lambda \neq \emptyset$. Then

$$
\delta_{2}(\Lambda, \emptyset)<\epsilon \Rightarrow \Lambda \subset \mathbb{R}^{n} \backslash B\left(0, \frac{1-\epsilon}{2 \epsilon}\right) .
$$

Proof. Let us assume the existence of $\lambda \in \Lambda$ such that $\|\lambda\| \leq \frac{1-\epsilon}{2 \epsilon}$ and let us show that this hypothesis implies that the assertion $\delta_{2}(\Lambda, \emptyset)<\epsilon$ is wrong. Take $D=\{\lambda\}$ and $E$ a dense subset in $\left(0, \frac{1}{2}\right)$. Each $\mathcal{B}_{m}$ in $\mathcal{B}_{(D, E)}$ is a set constituted by only one ball: say the ball $\stackrel{\circ}{B}\left(\lambda, e_{m}\right)$ with $e_{m} \in E$. We deduce that $\phi_{\mathcal{B}_{m}}(\Lambda)=e_{m}$. Since $\phi_{\mathcal{B}_{m}}(\emptyset)=0$, the following inequality holds:

$$
d_{\lambda,(D, E)}(\Lambda, \emptyset)=\sup _{m} \frac{e_{m}}{1 / 2+\|\lambda\|}=\frac{1 / 2}{1 / 2+\|\lambda\|} \leq \delta_{2}(\Lambda, \emptyset) .
$$

But $\epsilon \leq \frac{1}{1+2\|\lambda\|}$ is equivalent to $\|\lambda\| \leq \frac{1-\epsilon}{2 \epsilon}$. Hence, $\epsilon \leq d_{\lambda,(D, E)}(\Lambda, \emptyset)$. We deduce $\delta_{2}(\Lambda, \emptyset) \geq \epsilon$ as claimed.

From Proposition 3.6 and Proposition 3.7 we deduce

Corollary 3.2. For all $t>0$ the Hausdorff metric $\Delta$ on $\mathcal{U D}(B(0, t),\|\cdot\|)$ is compatible with the restriction of the topology of $\left(\mathcal{U D}, \delta_{2}\right)$ to the space $\mathcal{U D}(B(0, t),\|\cdot\|)_{f}=\mathcal{U D}(B(0, t),\|\cdot\|)$.

The converse of Proposition 3.7 is much harder (see Appendix).

3.3. Topological equivalence and point-removal. The "point-removal process" of a subcollection of points of a $\mathcal{U D}$-set is particularly easy to describe with $d$ and $\delta_{2}$. For all $\Lambda \in \mathcal{U D}$ and $R>0$, denote by $\Lambda_{R}$ the new $\mathcal{U} \mathcal{D}$-set $\Lambda \cap \stackrel{\circ}{B}(0, R)$.

Proposition 3.8. Let $\delta=d$ or $\delta_{2}$. Let $\Lambda, \Lambda^{\prime} \in \mathcal{U D}$ and $C$ be an arbitrary subset of $\Lambda \cap \Lambda^{\prime}$. Then $\delta\left(\Lambda, \Lambda^{\prime}\right)=\delta\left(\Lambda \backslash C, \Lambda^{\prime} \backslash C\right)$. In particular, for all $R>0$,

$$
\delta\left(\Lambda, \Lambda^{\prime}\right)=\delta\left(\Lambda \backslash\left(\Lambda \cap \Lambda^{\prime}\right), \Lambda^{\prime} \backslash\left(\Lambda \cap \Lambda^{\prime}\right)\right) \text { and } \delta\left(\Lambda \backslash \Lambda_{R}, \emptyset\right)=\delta\left(\Lambda_{R}, \Lambda\right) .
$$

Proof. These results follow readily from the definitions of $d$ and $\delta_{2}$. 
Remark. The distance (for $\delta_{2}$ ) between two dense (equal) sphere packings (radius $\frac{1}{2}$ ) differing only by a small cluster of spheres lying about the point $z$ is exactly the distance between these two clusters, say $\mathcal{C}_{1}$ and $\mathcal{C}_{2}$. Thus it is very easy to see that it is a ratio (from (3.4) in Section 3.2.1) roughly given by $\frac{m}{1 / 2+\|z\|} f\left(\mathcal{C}_{1}, \mathcal{C}_{2}\right)$, when $\|z\|$ is large enough, where $m$ is the (common) number of spheres of $\mathcal{C}_{1}$ (or $\mathcal{C}_{2}$ ) and $f\left(\mathcal{C}_{1}, \mathcal{C}_{2}\right.$ ) a function which depends upon the relative positions of the spheres inside $\mathcal{C}_{1}$ and $\mathcal{C}_{2}$. Such simple expressions are easy to handle, can be made more precise and can be differentiated to study optimal positioning of clusters in dense sphere packings [MVG1].

Let us show that the metrics $d, \delta_{1}, \delta_{2}$ are topologically equivalent on $\mathcal{U D}$.

Proposition 3.9. For any averaging sequence $\left(K_{i}\right)_{i \geq 1}$ of compact sets of $\mathbb{R}^{n}$ which contains the base point 0 , the metric d associated with it is such that:

(i) $d$ and $\delta_{1}$ are topologically equivalent,

(ii) $d$ and $\delta_{2}$ are topologically equivalent.

Proof. It suffices to show that the identity map is bicontinuous in each case.

(i) $\left(\mathcal{U D}, \delta_{1}\right) \stackrel{\text { id }}{\rightarrow}(\mathcal{U D}, d)$ is continuous: let $\epsilon>0$ be small enough and assume $\Lambda, \Lambda^{\prime} \in \mathcal{U D}$ with $\Lambda \neq \emptyset$. Let $\eta \in(0,1)$. Let $k$ be the greatest integer such that $K_{k} \subset B\left(0, \frac{\lambda}{R_{\min } \eta}\right)$. The map $\eta \rightarrow 2^{-k}$ takes the value 0 at zero and is continuous at zero. Then there exists $\eta_{0}$ such that $\eta \leq \eta_{0} \Rightarrow 2^{-k} \leq \epsilon / 2$. Now, if $\delta_{1}\left(\Lambda, \Lambda^{\prime}\right)<\eta$, then $R_{\min } \eta \in \Omega\left(\Lambda, \Lambda^{\prime}\right)$ and $\Delta\left(\Lambda \cap B\left(0, \frac{\lambda}{R_{\min } \eta}\right), \Lambda^{\prime} \cap B\left(0, \frac{\lambda}{R_{\min } \eta}\right)\right) \leq R_{\min } \eta$. We deduce:

$$
d\left(\Lambda, \Lambda^{\prime}\right) \leq \sum_{i=1}^{k} 2^{-i} R_{\min } \eta+\sum_{i \geq k+1} 2^{-i} \leq R_{\min } \eta+2^{-k} .
$$

Hence, for all $\eta<\min \left\{\frac{\epsilon}{2 R_{\min }}, \eta_{0}\right\}$ the inequality $\delta_{1}\left(\Lambda, \Lambda^{\prime}\right)<\eta$ implies $d\left(\Lambda, \Lambda^{\prime}\right)<\epsilon / 2+\epsilon / 2=\epsilon$, hence the claim. Assume now $\Lambda=\emptyset$. Given $\epsilon>0$ small enough, by the definition of $d$, there exists $R>0$ such that $d\left(\emptyset, \Lambda^{\prime}\right)<\epsilon$ for all $\Lambda^{\prime} \subset \mathbb{R}^{n} \backslash B(0, R)$. Take $\eta=\frac{\lambda}{R R_{\min }}$. Then the inequality $\delta_{1}\left(\emptyset, \Lambda^{\prime}\right)<\eta$ implies $R_{\min } \eta \in \Omega\left(\emptyset, \Lambda^{\prime}\right)$, hence $\Lambda^{\prime} \cap B\left(0, \frac{\lambda}{R_{\min } \eta}\right)=$ $\Lambda^{\prime} \cap B(0, R)=\emptyset$. We deduce: $\delta_{1}\left(\emptyset, \Lambda^{\prime}\right)<\eta \Rightarrow d\left(\emptyset, \Lambda^{\prime}\right)<\epsilon$, hence the continuity at $\emptyset$.

$(\mathcal{U D}, d) \stackrel{\text { id }}{\rightarrow}\left(\mathcal{U D}, \delta_{1}\right)$ is continuous: let $\epsilon>0$ be small enough and assume $\Lambda \neq \emptyset$. By Proposition 2.2 there exists $\eta$ such that:

$$
\begin{aligned}
d\left(\Lambda \cap B\left(0, \frac{\lambda}{R_{\min } \epsilon}\right), \Lambda^{\prime}\right. & \left.\cap B\left(0, \frac{\lambda}{R_{\min } \epsilon}\right)\right)<\eta \\
& \Rightarrow \Delta\left(\Lambda \cap B\left(0, \frac{\lambda}{R_{\min } \epsilon}\right), \Lambda^{\prime} \cap B\left(0, \frac{\lambda}{R_{\min } \epsilon}\right)\right)<R_{\min } \epsilon .
\end{aligned}
$$

Since

$$
d\left(\Lambda \cap B\left(0, \frac{\lambda}{R_{\min } \epsilon}\right), \Lambda^{\prime} \cap B\left(0, \frac{\lambda}{R_{\min } \epsilon}\right)\right) \leq d\left(\Lambda, \Lambda^{\prime}\right)
$$


and that

$$
\Delta\left(\Lambda \cap B\left(0, \frac{\lambda}{R_{\min } \epsilon}\right), \Lambda^{\prime} \cap B\left(0, \frac{\lambda}{R_{\min } \epsilon}\right)\right) \leq R_{\min } \epsilon
$$

is equivalent to $\delta_{1}\left(\Lambda, \Lambda^{\prime}\right)<\epsilon$, we have: $d\left(\Lambda, \Lambda^{\prime}\right)<\eta \Rightarrow \delta_{1}\left(\Lambda, \Lambda^{\prime}\right)<\epsilon$, hence the claim. Assume now $\Lambda=\emptyset$. Let $\epsilon>0$. Let $j$ the smallest integer such that $B\left(0, \frac{\lambda}{R_{\min \epsilon}}\right) \subset K_{j}$. We have $d\left(\emptyset, \Lambda^{\prime}\right)=2^{-u+1}$ where $u$ is the smallest integer such that $\Lambda^{\prime} \cap K_{u} \neq \emptyset$, so that $\Lambda^{\prime} \cap K_{i}=\emptyset$ for $i=1,2, \ldots, u-1$. Then we choose $\eta \in(0,1)$ small enough such that $d\left(\emptyset, \Lambda^{\prime}\right)=2^{-u+1}<\eta<2^{-u+2}$ with $u>j$. Hence $\Lambda^{\prime} \cap K_{j}=\emptyset$. Then $\Lambda^{\prime} \subset \mathbb{R}^{n} \backslash B\left(0, \frac{\lambda}{R_{\min } \epsilon}\right)$. We deduce $\delta_{1}\left(\emptyset, \Lambda^{\prime}\right)<\epsilon$ and the continuity at $\emptyset$.

(ii) $\left(\mathcal{U D}, \delta_{2}\right) \stackrel{\text { id }}{\rightarrow}(\mathcal{U D}, d)$ is continuous: let us first assume that the condition $\sum_{i \geq 1} 2^{-i} \mathcal{R}_{\mathcal{K}_{i}}<+\infty$ holds. Let $\epsilon>0$ be small enough. Let $\eta \in(0,1)$ and assume $\delta_{2}\left(\Lambda, \Lambda^{\prime}\right)<\eta$ where $\Lambda \neq \emptyset$. Define $k=k(\eta)$ by the conditions: $K_{k} \subset B\left(0, \frac{1-\eta}{2 \eta}\right)$ and $K_{k+1} \not \subset B\left(0, \frac{1-\eta}{2 \eta}\right)$. Since the map $\eta \rightarrow 2^{-k}$ takes the value 0 at zero and is continuous at zero, there exists $\eta_{0}$ such that $\eta \leq \eta_{0} \Rightarrow 2^{-k} \leq \epsilon / 2$. On the other hand, for all $i$ such that $K_{i} \subset B\left(0, \frac{1-\eta}{2 \eta}\right), \Delta\left(\Lambda \cap K_{i}, \Lambda^{\prime} \cap K_{i}\right)=d_{i}\left(\Lambda, \Lambda^{\prime}\right) \leq\left(\frac{1}{2}+\mathcal{R}_{K_{i}}\right) \eta$ by Proposition 3.6. Then

$$
d\left(\Lambda, \Lambda^{\prime}\right) \leq \sum_{i=1}^{k} 2^{-i}\left(\frac{1}{2}+\mathcal{R}_{K_{i}}\right) \eta+\sum_{i \geq k+1} 2^{-i} \leq\left(\frac{1}{2}+\sum_{i \geq 1} 2^{-i} \mathcal{R}_{K_{i}}\right) \eta+2^{-k} .
$$

There exists $\eta_{1}$ such that $\eta \leq \eta_{1} \Rightarrow\left(\frac{1}{2}+\sum_{i \geq 1} 2^{-i} \mathcal{R}_{\left(K_{i}\right.}\right) \eta \leq \epsilon / 2$. Then, for $\eta \leq \min \left\{\eta_{0}, \eta_{1}\right\}$, we have $d\left(\Lambda, \Lambda^{\prime}\right) \leq \epsilon / 2+\epsilon / 2=\epsilon$. This proves the continuity at all $\Lambda \neq \emptyset$. Continuity at $\emptyset$ arises readily from the definition of $d$ and Proposition 3.7. Using the Remark in Section 2, we wee that the condition $\sum_{i>1} 2^{-i} \mathcal{R}_{\mathcal{K}_{i}}<+\infty$ can be removed. Thus we obtain the claim in full generality.

$(\mathcal{U D}, d) \stackrel{\text { id }}{\rightarrow}\left(\mathcal{U D}, \delta_{2}\right)$ is continuous: let $\epsilon>0$ be small enough and assume $\Lambda \neq \emptyset$. By Proposition A.1 there exists $R$ such that

$$
\delta_{2}\left(\Lambda, \Lambda_{R}\right)=\delta_{2}\left(\Lambda \backslash \Lambda_{R}, \emptyset\right) \leq \epsilon / 3 \text { and } \delta_{2}\left(\Lambda^{\prime}, \Lambda_{R}^{\prime}\right)=\delta_{2}\left(\Lambda^{\prime} \backslash \Lambda_{R}^{\prime}, \emptyset\right) \leq \epsilon / 3 \text {. }
$$

By Corollary 3.2 there exists $\eta_{0}$ such that

$$
\Delta\left(\Lambda_{R}, \Lambda_{R}^{\prime}\right)<\eta_{0} \Rightarrow \delta_{2}\left(\Lambda_{R}, \Lambda_{R}^{\prime}\right)<\epsilon / 3 .
$$

Let $j \geq 1$ be the smallest integer such that $B(0, R) \subset K_{j}$. Let us take $\eta \in\left(0,2^{-j}\right)$ such that $\frac{\eta}{2^{-j}-\eta}<\eta_{0} \Longleftrightarrow \eta<\frac{\eta_{0} 2^{-j}}{1+\eta_{0}}$. Then, $d\left(\Lambda, \Lambda^{\prime}\right)=\sum_{i \geq 1} \frac{2^{-i} d_{i}\left(\Lambda, \Lambda^{\prime}\right)}{1+d_{i}\left(\Lambda, \Lambda^{\prime}\right)}<\eta \Rightarrow \frac{2^{-i} d_{i}\left(\Lambda, \Lambda^{\prime}\right)}{1+d_{i}\left(\Lambda, \Lambda^{\prime}\right)}<\eta$ for all $i=1,2, \ldots, j$. 
We deduce $\Delta\left(\Lambda_{R}, \Lambda_{R}^{\prime}\right) \leq d_{j}\left(\Lambda, \Lambda^{\prime}\right)=\Delta\left(\Lambda \cap K_{j}, \Lambda^{\prime} \cap K_{j}\right)<\frac{\eta}{2^{-i}-\eta}<\eta_{0}$. Thus

$$
\delta_{2}\left(\Lambda, \Lambda^{\prime}\right) \leq \delta_{2}\left(\Lambda, \Lambda_{R}\right)+\delta_{2}\left(\Lambda_{R}, \Lambda_{R}^{\prime}\right)+\delta_{2}\left(\Lambda_{R}^{\prime}, \Lambda^{\prime}\right) \leq \epsilon / 3+\epsilon / 3+\epsilon / 3=\epsilon .
$$

We deduce the claim for $\Lambda \neq \emptyset$. Assume now $\Lambda=\emptyset$. Let $\epsilon>0$ be small enough. By Proposition A.1 there exists $R$ such that $\Lambda^{\prime} \subset \mathbb{R}^{n} \backslash B(0, R)$ implies $\delta_{2}\left(\Lambda^{\prime}, \emptyset\right) \leq \epsilon$. Let $j$ be the smallest integer such that $\Lambda^{\prime} \cap K_{j} \neq \emptyset$. Then $\Lambda^{\prime} \cap K_{j-1}=\emptyset$ and $B(0, R) \subset K_{j-1}$. Since $d\left(\emptyset, \Lambda^{\prime}\right)=\sum_{i \geq j} 2^{-i}=$ $2^{-j+1}$ (with $\Lambda^{\prime} \neq \emptyset$ ) we take $\eta$ such that $2^{-j+1}<\eta<2^{-j+2}$, for instance $\eta=3.2^{-j}$. Then $d\left(\emptyset, \Lambda^{\prime}\right)<\eta \Rightarrow \delta_{2}\left(\Lambda^{\prime}, \emptyset\right) \leq \epsilon$, hence the continuity at $\emptyset$.

The following proposition is fundamental. It shows the uniform continuity of the point-removal process at infinity.

Proposition 3.10. Let $\Lambda \in \mathcal{U D}$. Denote by $\delta$ either $d$ or $\delta_{1}$. Then

$$
\lim _{R \rightarrow \infty} \delta\left(\Lambda, \Lambda_{R}\right)=\lim _{R \rightarrow \infty} \delta\left(\Lambda \backslash \Lambda_{R}, \emptyset\right)=0 .
$$

Moreover the convergence is uniform in the following sense:

$$
\forall \epsilon \in(0,1), \exists R>0 \text { such that: } \Lambda \subset \mathbb{R}^{n} \backslash B(0, R) \Rightarrow \delta(\Lambda, \emptyset)<\epsilon .
$$

Proof. If $\Lambda$ is finite, the limit is obviously zero. Assume $\Lambda$ infinite. The claim is obvious when $\delta=d$ or $\delta=\delta_{1}$ by definition of $d$ and $\delta_{1}$.

Remark. A direct (and self-contained) proof of Proposition 3.10 with $\delta=\delta_{2}$ can be found in the Appendix (Proposition A.1).

Corollary 3.3. The subset $\mathcal{U} \mathcal{D}_{f}$ is dense in $\mathcal{U D}$.

\section{Proof of Theorem 1.2}

Let $\left(K_{k}\right)_{k \geq 1}$ be an averaging sequence of compact sets which contains the base point 0 and $d$ the metric associated with it. Let us embedd $\mathcal{U D}$ in the product space

$$
\mathcal{U D} \subset \prod_{k \geq 1} \mathcal{U D}\left(K_{k},\|\cdot\|\right)_{1}=W
$$

each $\mathcal{U D}\left(K_{k},\|\cdot\|\right)_{1}$ being a compact metric space with the Hausdorff metric $\Delta$, equivalently with $d, \delta_{1}$ or $\delta_{2}$ by Proposition 2.1, resp. Proposition 3.1, or resp. Corollary 3.2. Thus the space $W$ is naturally a compact space by Tychonov's Theorem, and it is clear that $\mathcal{U D}$ is closed inside. Indeed, the image can be identified with the families $\left(V_{k}\right) \in \mathcal{U D}\left(K_{k},\|\cdot\|\right)_{1}$ such that $V_{k} \cap K_{k-1}=V_{k-1}$. This is a special case of a projective limit. Therefore it is compact.

For all $R>0$ the subspace $X_{R}$ of the Delone sets of constant $R$ is closed in $(\mathcal{U D}, d)$, since the relative denseness conditions are closed. Nevertheless, 
let us prove directly this result using $\delta_{2}$. Let $\Lambda \in \mathcal{U D} \backslash X_{R}$. We will show that it is contained in an open subset disjoint from $X_{R}$ that will prove that $X_{R}$ is closed. Since $\Lambda \notin X_{R}$, there exists $z \in \mathbb{R}^{n}$ such that $\|z-\lambda\|>R$ for all $\lambda \in \Lambda$. Let $l=\operatorname{dist}(\{z\}, \Lambda)>R$. Denote $\Lambda-z:=\{\lambda-z \mid \lambda \in \Lambda\}$ the translated set. For $\epsilon>0$ small enough and all $\Gamma$ in the open $\delta_{2}$-ball $\left\{\Omega \in \mathcal{U D} \mid \delta_{2}(\Omega, \Lambda-z)<\epsilon\right\}$, all the elements $\gamma$ of $\Gamma$ satisfy the inequality: $\|\gamma\| \geq R+\frac{l-R}{2}>R$ by the point pairing property (Proposition 3.6); all these point sets $\Gamma$ are outside $X_{R}$. Since the translation by $z$ is bicontinuous the $\mathcal{U D}$-set $\Lambda$ is contained in the open subset $z+\left\{\Omega \in \mathcal{U D} \mid \delta_{2}(\Omega, \Lambda-z)<\epsilon\right\}$ which is disjoint of $X_{R}$.

\section{Theorem 1.2 implies Theorem 1.1}

Let $\mathcal{L}_{n}$ be the space of lattices in $\mathbb{R}^{n}$, identified with the locally compact homogeneous space $G L(n, \mathbb{R}) / G L(n, \mathbb{Z})[\mathrm{GL}],[\mathrm{Ca}]$ (Recall that a lattice in $\mathbb{R}^{n}$ is a discrete $\mathbb{Z}$-module of maximal rank of $\mathbb{R}^{n}$, equivalently a discrete subgroup of the group of translations of $\mathbb{R}^{n}$ with compact fundamental region). The following proposition is a key result for proving Theorem 1.1 from Theorem 1.2, using $\delta_{2}$ for the proof and invoking Proposition 3.9 for the other metrics.

Proposition 5.1. The restriction of the metric $\delta_{2}$, resp. $d$ or $\delta_{1}$, to $\mathcal{L}_{n} \cap \mathcal{U D} \subset \mathcal{U D}$ is compatible with the topology on $\mathcal{L}_{n} \cap \mathcal{U D}$ induced by the quotient topology of $\mathcal{L}_{n}=G L(n, \mathbb{R}) / G L(n, \mathbb{Z})$.

Proof. This proposition is a reformulation of the following proposition.

Proposition 5.2. Let $L \in \mathcal{L}_{n} \cap \mathcal{U D}$. Denote by $\left\{e_{1}, e_{2}, \ldots, e_{n}\right\}$ a basis of $L$. Then

(i) for all $\epsilon>0$ small enough there exists $\eta>0$ such that any $\mathbb{Z}$-module $L^{\prime} \in \mathcal{U D}$ contained in the open ball $\left\{\Lambda \in \mathcal{U D} \mid \delta_{2}(L, \Lambda)<\eta\right\}$ is of rank $n$ and admits a basis $\left\{e_{1}^{\prime}, e_{2}^{\prime}, \ldots, e_{n}^{\prime}\right\}$ which satisfies the property: $\max _{i=1,2, \ldots, n}\left\|e_{i}-e_{i}^{\prime}\right\|<\epsilon$;

(ii) $\forall \eta \in(0,1), \exists \epsilon>0$ such that any lattice $L^{\prime} \in \mathcal{U D}$ of $\mathbb{R}^{n}$ admitting a basis $\left\{e_{1}^{\prime}, e_{2}^{\prime}, \ldots, e_{n}^{\prime}\right\}$ which satisfies $\max _{i=1,2, \ldots, n}\left\|e_{i}-e_{i}^{\prime}\right\|<\epsilon$ is such that $\delta_{2}\left(L, L^{\prime}\right)<\eta$.

Proof. (i) First let us chose $\epsilon_{0}>0$ small enough such that any $n$-tuple $\left\{a_{1}, a_{2}, \ldots, a_{n}\right\}$ of points of $\mathbb{R}^{n}$ with $a_{i} \in B\left(e_{i}, \epsilon_{0}\right), i=1,2, \ldots, n$, is such that the vectors $\left\{O a_{1}, O a_{2}, \ldots, O a_{n}\right\}$ are $\mathbb{Z}$-linearly independant (as usual we identify the point $a_{i}$ with the vector $\left.O a_{i}, i=1,2, \ldots, n\right)$. For instance, let us take $\epsilon_{0}=\frac{1}{3} \min _{i=1,2, \ldots, n}\left\{\operatorname{dist}\left(\left\{e_{i}\right\}\right.\right.$, Vect $\left.\left._{i}\right)\right\}$, if $\operatorname{Vect}_{i}, i=1,2, \ldots, n$, is the $\mathbb{R}$-span generated by the vectors $O e_{1}, O e_{2}, \ldots, O e_{i-1}, O e_{i+1}, \ldots, O e_{n}$. Let $\epsilon \in\left(0, \epsilon_{0}\right)$. 
Assume that $\Lambda$ is a $\mathcal{U D}$-set such that $\delta_{2}(L, \Lambda)<\eta$ with $\eta$ small enough. By Proposition 3.6 a pairing between the points of $L$ and $\Lambda$ occurs over a certain distance, which is $\frac{1-\eta}{2 \eta}$, from the origin. Let us take $\eta_{1}$ small enough in order to have $\frac{1-\eta_{1}}{2 \eta_{1}} \geq \max _{i=1,2, \ldots, n}\left\|e_{i}\right\|$. From Proposition 3.6 the condition $0<\eta<\eta_{1}$ implies the existence of $n$ points $e_{1}^{\prime}, e_{2}^{\prime}, \ldots, e_{n}^{\prime}$ in $\Lambda$, the respective close-neighbours of the points $e_{1}, e_{2}, \ldots, e_{n}$ of $L$, which satisfy $\left\|e_{i}^{\prime}-e_{i}\right\| \leq\left(\frac{1}{2}+\left\|e_{i}\right\|\right) \eta$ for $i=1,2, \ldots, n$. Take $\eta<\eta_{1}$ such that $\left(\frac{1}{2}+\max _{i=1,2, \ldots, n}\left\|e_{i}\right\|\right) \eta<\epsilon$. Since $\epsilon<\epsilon_{0}$, the vectors $O e_{1}^{\prime}, O e_{2}^{\prime}, \ldots, O e_{n}^{\prime}$ are $\mathbb{Z}$-linearly independant. This means that if $\Lambda \in \mathcal{U D}$ is a $\mathbb{Z}$-module of $\mathbb{R}^{n}$ (necessarily discrete) which satisfies $\delta_{2}(L, \Lambda)<\eta, \Lambda$ is necessarily of rank $n$ and contains the lattice $\sum_{i=1}^{n} \mathbb{Z} e_{i}^{\prime}$. Let us show that there is equality. Denote by $\mathcal{V}^{\prime}=\left\{\sum_{i=1}^{n} \theta_{i} e_{i}^{\prime} \mid 0 \leq \theta_{i}<1\right.$ for all $\left.i=1,2, \ldots, n\right\}$. The adherence $\overline{\mathcal{V}^{\prime}}$ of $\mathcal{V}^{\prime}$ contains only the points $\sum_{i=1}^{n} j_{i} e_{i}^{\prime}$ of $\Lambda$, with $j_{i}=0$ or 1 , by the property of the pairing (Proposition 3.6). Therefore the free system $\left\{O e_{1}^{\prime}, O e_{2}^{\prime}, \ldots, O e_{n}^{\prime}\right\}$ is a basis of $\Lambda$.

(ii) Conversely, let $0<\eta<1$ and $L^{\prime} \in \mathcal{U D} \cap \mathcal{L}_{n}$. For all $R>0$ the inequality $\delta_{2}\left(L, L^{\prime}\right) \leq \delta_{2}\left(L, L_{R}\right)+\delta_{2}\left(L_{R}, L_{R}^{\prime}\right)+\delta_{2}\left(L_{R}^{\prime}, L^{\prime}\right)$ holds. By Proposition A.1 let us take $R$ large enough such that $\delta_{2}\left(L, L_{R}\right)<\eta / 3$ and $\delta_{2}\left(L^{\prime}, L_{R}^{\prime}\right)<\eta / 3$. Let us now show that, if $L^{\prime}$ admits a basis $\left\{e_{1}^{\prime}, e_{2}^{\prime}, \ldots, e_{n}^{\prime}\right\}$ which satisfies $\max _{i=1,2, \ldots, n}\left\|e_{i}-e_{i}^{\prime}\right\|<\epsilon$, then $\epsilon$ can be taken small enough to have $\delta_{2}\left(L_{R}, L_{R}^{\prime}\right)<\eta / 3$ ( $R$ kept fixed). Indeed, $L_{R}$ and $L_{R}^{\prime}$ are finite $\mathcal{U D}$-sets. Denote $N:=\# L_{R}$. For all $\alpha \in \mathbb{R}^{n}$, all $(D, E) \in \mathcal{E}$ and all $\mathcal{B}_{m} \in \mathcal{B}_{(D, E)}$, by continuity of the function $f$, the mapping

$$
\left(x_{1}, x_{2}, \ldots, x_{N}\right) \rightarrow \phi_{\mathcal{B}_{m}}\left(\left\{x_{1}, x_{2}, \ldots, x_{N}\right\}\right):=\sum_{\stackrel{\circ}{B}(c, \omega) \in \mathcal{B}_{m}} \sum_{i=1}^{N} \omega f\left(\frac{x_{i}-c}{\omega}\right)
$$

is continuous on $B(0, R)^{N}$ for the standard product topology. Therefore all the mappings $d_{\alpha,(D, E)}\left(L_{R}, \cdot\right)$ :

$$
\left(x_{1}, x_{2}, \ldots, x_{N}\right) \rightarrow \sup _{\mathcal{B}_{m}} \frac{\left|\phi_{\mathcal{B}_{m}}\left(L_{R}\right)-\phi_{\mathcal{B}_{m}}\left(\left\{x_{1}, x_{2}, \ldots, x_{N}\right\}\right)\right|}{\left(\frac{1}{2}+\|\alpha\|+\left\|\alpha-c_{j_{1}}\right\|+\left\|\alpha-c_{j_{2}}\right\|+\cdots+\left\|\alpha-c_{j_{N}}\right\|\right)}
$$

are continuous on $B(0, R)^{N}$. The map

$$
\left(x_{1}, x_{2}, \ldots, x_{N}\right) \rightarrow d\left(L_{R},\left\{x_{1}, x_{2}, \ldots, x_{N}\right\}\right)
$$

is then continuous on $B(0, R)^{N}$. Take for $\left\{x_{1}, x_{2}, \ldots, x_{N}\right\}$ the point set $L_{R}^{\prime}$. Consequently the quantity $\delta_{2}\left(L_{R}, L_{R}^{\prime}\right)$ is strictly less than $\eta / 3$ as soon as $\epsilon$ is small enough. Finally $\delta_{2}\left(L, L^{\prime}\right)<3 \eta / 3=\eta$ and we deduce the claim.

Recall that if $L$ is a lattice in $\mathbb{R}^{n}$ and $A$ a basis of $L$, then $|\operatorname{det}(A)|$ is called the determinant of $L$; we will denote it by $|L|$. It is the volume of its fundamental region. 
Proposition 5.3. The subspace $\left\{L \in \mathcal{U D} \cap \mathcal{L}_{n}|0<| L \mid \leq M\right\} \subset \mathcal{L}_{n} \cap \mathcal{U D}$ is compact for all $M>0$.

Proof. By Proposition 5.1 and since $(\mathcal{U D}, d)$ is a compact topological space, we have just to show that $\left\{L \in \mathcal{U D} \cap \mathcal{L}_{n}|0<| L \mid \leq M\right\}$ is closed. Since the operations $x+y$ and $x y$ are continuous, the determinant function $|\cdot|$ is continuous on $\mathcal{L}_{n}$. Hence $\left\{L \in \mathcal{U D} \cap \mathcal{L}_{n}|| L \mid>M\right\}=|\cdot|^{-1}((M,+\infty))$ is an open set as reciprocal image of the open interval $(M,+\infty)$ by the continuous application $|\cdot|$. By taking its complementary subspace in $\mathcal{U D} \cap \mathcal{L}_{n}$ we deduce the claim.

Let us now prove Theorem 1.1. Let us consider a sequence of lattices ( $\left.L_{r}\right)$ of $\mathbb{R}^{n}$ such that: (i) $\|x\| \geq 1$ for all $x \in L_{r}, x \neq 0$, (ii) the determinant $\left|L_{r}\right|$ of $L_{r}$ satisfies $\left|L_{r}\right| \leq M$ with $M$ a constant $<+\infty$ independent of $r$. Then $L_{r} \in\left\{L \in \mathcal{U D} \cap \mathcal{L}_{n}|0<| L \mid \leq M\right\}$, for all $r$, which is compact by Proposition 5.3. Then, by the Bolzano-Weierstrass property, one can extract from the sequence $\left(L_{r}\right)$ a subsequence $\left(L_{r^{\prime}}\right)$ that converges to a lattice $L$ of $\mathbb{R}^{n}$. By continuity of the determinant function $|\cdot|$ and Proposition 5.1, we obtain: $|L|=\lim _{r^{\prime} \rightarrow+\infty}\left|L_{r^{\prime}}\right|$. This concludes the proof.

\section{Arbitrary metric spaces}

The topological space $\left(\mathcal{U D}\left(\mathbb{R}^{n},\|\cdot\|\right)_{1}, d\right)$ is a Polish space [B], but its topology is not classical. It is routine to compare it with the topologies reviewed by Kelley [Ke] and Michael [Mi] on spaces of nonempty closed subsets of $\mathbb{R}^{n}$ and to conclude that it is none of them (see also $\S 4$ in [BL], and [Bo]). The metric space $\cup_{r>0} \mathcal{U D}\left(\mathbb{R}^{n},\|\cdot\|\right)_{r, f}$ is dense in $\mathcal{D}_{l f}\left(\mathbb{R}^{n},\|\cdot\|\right)$. The metric space $\cup_{r>0} \mathcal{U} \mathcal{D}\left(\mathbb{R}^{n},\|\cdot\|\right)_{r}$, endowed with $d$ or $\delta_{1}$, is not compact and is much bigger than the space of lattices of $\mathbb{R}^{n}$. For instance, if $n=1$, it contains all the Meyer sets $\mathbb{Z}_{\beta}$ of $\beta$-integers (integers in base $\beta$ ) where $\beta$ is a Pisot number or a Parry number [GVG] (see [Mo], [MVG] for a modern language on Meyer sets and Delone sets).

Let $(H, \delta)$ be a metric space.

Proposition 6.1. If $\operatorname{diam}(H)=+\infty$, then $H$ only contains infinite Delone sets.

Proof. Assume that $\Lambda=\left\{\lambda_{1}, \lambda_{2}, \ldots, \lambda_{I}\right\}, 1 \leq I<+\infty$, is a finite Delone set in $H$ of constants $(r, R)$ and let us show the contradiction. Since, for all $z \in H$, there exists $\lambda_{i} \in \Lambda$ such that $\lambda_{i} \in z+B(0, R)$, then $z \in B\left(\lambda_{i}, R\right)$ and we would have $H \subset \cup_{i=1}^{I} B\left(\lambda_{i}, R\right)$ which is of diameter less than $2 R I$. Thus we would have $\operatorname{diam}(H) \leq 2 R I<+\infty$. Contradiction.

If $H$ is compact, then $\operatorname{diam}(H)<+\infty$ and all the Delone sets of $H$ are finite. For all $r>\operatorname{diam}(H)$, the uniformly discrete subsets of $H$ of 
constant $r$ are empty. When $r \leq \operatorname{diam}(H)$ a uniformly discrete subset of $H$ of constant $r$ is either the empty set $\emptyset$ or is finite. Thus the set-theoretic equality holds:

$$
\bigcup_{0<r \leq \operatorname{diam}(H)} \mathcal{U D}(H, \delta)_{r} \backslash\{\emptyset\}=\bigcup_{\substack{0<R, 0<r \leq \operatorname{diam}(H)}} X(H, \delta)_{r, R} .
$$

This space endowed with the Hausdorff metric $\Delta$ is a compact space and $\emptyset$ is an isolated point. This provides positive answers to Question 1.1 and Question 1.2 with $d=\Delta$ since, for all $0<r \leq \operatorname{diam}(H)$ and $R>0$, $\mathcal{U D}(H, \delta)_{r}$ is closed in $\cup_{0<r \leq \operatorname{diam}(H)} \mathcal{U D}(H, \delta)_{r}$, and $X(H, \delta)_{r, R}$ is closed in $\cup_{0<R, 0<r \leq \operatorname{diam}(H)} X(H, \delta)_{r, R}$.

The following Theorem is an improvment of Macbeath and Swierczkowski's Theorem [MS] in the context of ("ambient") metric spaces, providing positive answers to Question 1.1 and Question 1.2.

Theorem 6.1. Let $(H, \delta)$ be a $\sigma$-compact and locally compact metric space for which $\operatorname{diam}(H)$ is infinite. Then, for all $r>0, \mathcal{U D}(H, \delta)_{r}$ can be endowed with a metric d such that the topological space $\left(\mathcal{U D}(H, \delta)_{r}, d\right)$ is compact and such that the Hausdorff metric on $\mathcal{U D}(H, \delta)_{r, f}$ is compatible with the restriction of the topology of $\left(\mathcal{U D}(H, \delta)_{r}, d\right)$ to $\mathcal{U D}(H, \delta)_{r, f}$. For all $R>0$, its subspace of the Delone sets of constants $(r, R)$ is closed.

Proof. The metric $d$ is the one constructed in Section 2 but now on $H$ instead of $\mathbb{R}^{n}$. From Section 4 we deduce the compactness of $\mathcal{U D}(H, \delta)_{r}$ for all $r>0$. Indeed, the proof in Section 4 is valid for all "ambient" metric spaces which are $\sigma$-compact and locally compact.

\section{Appendix A}

This Appendix gives a proof of Proposition A.1. Proposition A.1 is related to the rest of the paper by the fact that it implies the topological equivalence between $d, \delta_{1}$ and $\delta_{2}$ on $\mathcal{U D}$ (see proof of Proposition 3.9) and is used in the proof of Proposition 5.2. Though fairly long, the present computations are not necessary for many applications concerning the topological space $\mathcal{U D}$.

Proposition A.1. Let $\Lambda \in \mathcal{U} D$. Then

$$
\lim _{R \rightarrow \infty} \delta_{2}\left(\Lambda, \Lambda_{R}\right)=\lim _{R \rightarrow \infty} \delta_{2}\left(\Lambda \backslash \Lambda_{R}, \emptyset\right)=0 .
$$

Moreover the convergence is uniform in the following sense:

$$
\forall \epsilon \in(0,1), \exists R>0 \text { such that: } \Lambda \subset \mathbb{R}^{n} \backslash B(0, R) \Rightarrow \delta_{2}(\Lambda, \emptyset)<\epsilon .
$$


Proof. We assume that $\Lambda$ is infinite in the sequel since, when $\Lambda$ is finite, the limit is obviously zero. To prove this result we use Stolarsky's inequality [St] (recalled in Proposition A.2 without proof) which provides an (uniform) upper bound of $\delta_{2}\left(\Lambda, \Lambda_{R}\right)$. Then we explicitely compute this (uniform) upper bound by means of representations of integers as sums of squares (of integers) (see Grosswald [Gr] for a survey) (Steps 1 and 2). This type of computation provides uniform convergence.

Proposition A.2 (Stolarsky [St]). Let $u, v$ rational integers such that $u \geq v \geq 1$. Let $\left\{x_{1}, x_{2}, \ldots, x_{u}\right\}$ be a finite set of $u$ points of $\mathbb{R}^{n}$ and $\left\{y_{1}, y_{2}, \ldots, y_{v}\right\}$ be another finite set of $v$ points of $\mathbb{R}^{n}, n \geq 2$. Let us define $h(u, v)=1$ if $u=v, h(u, v)=\frac{u-1}{v}$ if $u>v$. Then

$$
\sum_{1 \leq i<j \leq u}\left\|x_{i}-x_{j}\right\|+\sum_{1 \leq i<j \leq v}\left\|y_{i}-y_{j}\right\| \leq h(u, v) \sum_{i=1}^{u} \sum_{j=1}^{v}\left\|x_{i}-y_{j}\right\|
$$

where the constant $h(u, v)$ is best possible.

Let us apply Proposition A.2. Take $v=1$ and $u=i_{m}+1 \geq 2$ with $x_{1}=0$ and $\left\|x_{i}\right\| \geq R$ for all $i=2,3, \ldots, u$; then put $y_{1}=\alpha \in \mathbb{R}^{n}$ arbitrary. The inequality (A.1) gives

$$
\sum_{j=2}^{i_{m}+1}\left\|x_{j}\right\|+\sum_{2 \leq i<j \leq i_{m}+1}\left\|x_{i}-x_{j}\right\| \leq h\left(i_{m}+1,1\right)\left(\|\alpha\|+\sum_{i=2}^{i_{m}+1}\left\|\alpha-x_{i}\right\|\right) .
$$

Consequently, setting $c_{i-1}=x_{i}$ for all $i=2,3, \ldots, i_{m}+1$ for keeping the notations as close as possible to the definition of $d_{\alpha,(D, E)}$ (see (3.4)), the following inequality holds:

$$
\frac{i_{m}}{1 / 2+\|\alpha\|+\sum_{i=1}^{i_{m}}\left\|\alpha-c_{i}\right\|} \leq \frac{i_{m}}{\frac{1}{2}+\frac{1}{i_{m}}\left(\sum_{j=1}^{i_{m}}\left\|c_{j}\right\|+\sum_{1 \leq i<j \leq i_{m}}\left\|c_{i}-c_{j}\right\|\right)} .
$$

The supremum of the right-hand side expression, over all possible configurations of balls in $\mathcal{B}_{(D, E)}$ and $(D, E) \in \mathcal{E}$ such that their centres $c_{i}$ satisfy $\left\|c_{i}\right\| \geq R$, is greater than $2 \delta_{2}\left(\Lambda \backslash \Lambda_{R}, \emptyset\right)$ (see Proposition 3.3 for the definition of $\delta_{2}$ ). We will show that it goes to zero when $R$ tends to infinity. For this, we will compute explicitely a lower bound of

$$
\eta\left(R, i_{m}\right):=\frac{1}{2 i_{m}}+\frac{1}{i_{m}^{2}} \sum_{j=1}^{i_{m}}\left\|c_{j}\right\|+\frac{1}{i_{m}^{2}} \sum_{1 \leq i<j \leq i_{m}}\left\|c_{i}-c_{j}\right\|
$$

as a function of $R$ and $i_{m}$, where $\eta\left(R, i_{m}\right)$ is the inverse of the right-hand side term in the inequality (A.2). In order to simplify the notations, we will study the quantity $\eta(R, m)$, what amounts merely to replace $m$ by $i_{m}$ in the rest of the proof for coming back to the inequality (A.2). 
We will proceed as follows, in three steps. The first step (Step 1) will consist in making this computation explicit when the points $c_{i}$ are on the lattice $\mathbb{Z}^{n}$ with $n \geq 5$. In other terms, we will prove:

$$
\lim _{R \rightarrow+\infty} \delta_{2}\left(\mathbb{Z}^{n}, \mathbb{Z}_{R}^{n}\right)=0 \text { for all } n \geq 5 .
$$

The second step (Step 2) will describe how to provide a lower bound of $\eta_{\Lambda}(R, m)$ (see its definition in Step 2) from $\eta(R, m)$ when the points $c_{i}$ are in a $\mathcal{U} \mathcal{D}$-set $\Lambda \subset \mathbb{R}^{n}$ which is not $\mathbb{Z}^{n}$ with still $n \geq 5$ for which the dimension of the $\mathbb{R}$-span of $\Lambda$ is $n$ or less than $n$. In other terms, we will prove:

$$
\lim _{R \rightarrow+\infty} \delta_{2}\left(\Lambda, \Lambda_{R}\right)=0 \quad \text { for all } \Lambda \in \mathcal{U D} \text { and } n \geq 5 \text {. }
$$

The final Step 3 will conclude when $n \in\{1,2,3,4\}$ making use of descent arguments to lower dimensions. In other terms, we will prove:

$$
\lim _{R \rightarrow+\infty} \delta_{2}\left(\Lambda, \Lambda_{R}\right)=0 \quad \text { for all } \Lambda \in \mathcal{U D} \text { and } n \leq 4
$$

Step 1.- Let us recall the assumptions: $R>\sqrt{2}$ (for technical reasons) and $c_{i} \in \mathbb{Z}^{n},\left\|c_{i}\right\| \geq R$, for all $i=1,2, \ldots, m$ with $i \neq j \Rightarrow c_{i} \neq c_{j}$. In order to find a lower bound of $\eta(R, m)$, we will compute a lower bound of $m^{-2} \sum_{1 \leq i<j \leq m}\left\|c_{i}-c_{j}\right\|(\mathrm{A} .5)$ and a lower bound of $m^{-2} \sum_{j=1}^{m}\left\|c_{j}\right\|$ (A.6) as a function of $R$ and $m$. These two bounds will be shown to be dependent (by (A.7) and (A.8)). The sum of these two lower bounds will present a minimum and the main difficulty will consist in showing that this minimum tends to infinity when $R$ tends to infinity.

Let us compute a lower bound of $m^{-2} \sum_{1 \leq i<j \leq m}\left\|c_{i}-c_{j}\right\|$. Let $s$ be a positive integer and consider the equation $s=\sum_{i=1}^{n} c_{q, i}^{2}$ with $c_{q, i} \in \mathbb{Z}$ for all $i=1,2, \ldots, n$. Any $n$-tuple $\left(c_{q, 1}, c_{q, 2}, \ldots, c_{q, n}\right)$ which satisfies this equation is called a solution of this equation. This solution represents the vector $c_{q}={ }^{t}\left(c_{q, 1}, c_{q, 2}, \ldots, c_{q, n}\right)$ in $\mathbb{Z}^{n}$ of norm $s^{1 / 2}$. Given $s$, denote by $r_{n}(s)$ the number of solutions of the above equation; it is the number of elements of $\mathbb{Z}^{n}$ which lie on the sphere $S(0, \sqrt{s})$ of centre the origin and radius $\sqrt{s}$. Obviously $r_{n}(0)=1, r_{n}(1)=2^{n}$. Now, for any integer $m>1$, there exists a unique integer $k$ such that

$$
\text { (A.3) } r_{n}(0)+r_{n}(1)+\cdots+r_{n}(k)<m \leq r_{n}(0)+r_{n}(1)+\cdots+r_{n}(k)+r_{n}(k+1)
$$

with $r_{n}(k) r_{n}(k+1) \neq 0$. We know (Grosswald [Gr], Chapters 9, 12 and 13) the behaviour of $r_{n}(s)$ when $n \geq 5$ : there exists two strictly positive constants $\widehat{K_{1}}(n)$ and $\widehat{K_{2}}(n)$ such that $r_{n}(s)=\rho_{n}(s)+O\left(s^{n / 4}\right)$ with $\widehat{K_{1}}(n) s^{n / 2-1} \leq \rho_{n}(s) \leq \widehat{K_{2}}(n) s^{n / 2-1}$ for any integer $s>0$. Therefore, there exists two strictly positive constants $K_{1}, K_{2}$, which depend upon $n$, such that $K_{2} \geq 1$ and $K_{1} s^{n / 2-1} \leq r_{n}(s) \leq K_{2} s^{n / 2-1}$ for any integer $s>0$. By 
saturating all the spheres $S\left(c_{1}, \sqrt{l}\right) \cap \mathbb{Z}^{n}$ for $l=0,1,2, \ldots, k$ we deduce

$$
\sum_{j=2}^{m}\left\|c_{j}-c_{1}\right\| \geq \sum_{j=2}^{r_{n}(0)+r_{n}(1)+\cdots+r_{n}(k)+1}\left\|c_{j}-c_{1}\right\| \geq \sum_{l=0}^{k} r_{n}(l) \sqrt{l}
$$

Let us consider that $m$ is equal to $r_{n}(0)+r_{n}(1)+\cdots+r_{n}(k)+r_{n}(k+1)$. We now proceed with the other sums $\sum_{j=i+1}^{m}\left\|c_{j}-c_{i}\right\|, i \geq 2$. For all $i=1,2, \ldots, r_{n}(k+1)$, the difference $m-i$ is greater than $r_{n}(0)+r_{n}(1)+$ $\cdots+r_{n}(k)$ and this implies

$$
\sum_{j=i+1}^{m}\left\|c_{j}-c_{i}\right\| \geq \sum_{l=0}^{k} r_{n}(l) \sqrt{l}
$$

Hence

$$
\sum_{i=1}^{r_{n}(k+1)} \sum_{j=i+1}^{m}\left\|c_{j}-c_{i}\right\| \geq r_{n}(k+1)\left(\sum_{l=0}^{k} r_{n}(l) \sqrt{l}\right)
$$

Since

$$
\begin{gathered}
\sum_{i=1}^{m-1} \sum_{j=i+1}^{m}\left\|c_{j}-c_{i}\right\|=\sum_{i=1}^{r_{n}(k+1)} \sum_{j=i+1}^{m}\left\|c_{j}-c_{i}\right\|+\sum_{i=r_{n}(k+1)+1}^{r_{n}(k+1)+r_{n}(k)} \sum_{j=i+1}^{m}\left\|c_{j}-c_{i}\right\|+ \\
\cdots+\sum_{i=r_{n}(k+1)+r_{n}(k)+\ldots+r_{n}(2)+1}^{r_{n}(k+1)+r_{n}(k)+\ldots+r_{n}(1)} \sum_{j=i+1}^{m}\left\|c_{j}-c_{i}\right\|,
\end{gathered}
$$

by reproducing the same computation term by term, we deduce

$$
\begin{aligned}
\sum_{i=1}^{m-1} \sum_{j=i+1}^{m}\left\|c_{j}-c_{i}\right\| & \geq r_{n}(k+1)\left(\sum_{l=0}^{k} r_{n}(l) \sqrt{l}\right) \\
& +r_{n}(k)\left(\sum_{l=0}^{k-1} r_{n}(l) \sqrt{l}\right)+\cdots+r_{n}(2) r_{n}(1)+2^{n} \\
& \geq \sum_{p=1}^{k+1} r_{n}(p)\left(\sum_{l=0}^{p-1} r_{n}(l) \sqrt{l}\right) \\
& \geq K_{1}^{2} \sum_{p=1}^{k+1} p^{\frac{n}{2}-1}\left(\sum_{l=0}^{p-1} l^{\frac{n-1}{2}}\right)
\end{aligned}
$$

Now make use of the following classical inequalities: for all $\beta>0$ and 
integer $r \geq 1,0+1^{\beta}+2^{\beta}+\cdots+(r-1)^{\beta} \leq \int_{0}^{r} x^{\beta} d x=\frac{r^{\beta+1}}{\beta+1} \leq 1^{\beta}+2^{\beta}+$ $\cdots+(r-1)^{\beta}+r^{\beta}$. We deduce the following inequalities

$$
\begin{aligned}
\sum_{i=1}^{m-1} \sum_{j=i+1}^{m}\left\|c_{j}-c_{i}\right\| & \geq \frac{2 K_{1}^{2}}{n+1} \sum_{p=1}^{k+1} p^{\frac{n}{2}-1}(p-1)^{\frac{n+1}{2}} \\
& \geq \frac{2 K_{1}^{2}}{n+1} \sum_{p=1}^{k+1}(p-1)^{\frac{n}{2}-1}(p-1)^{\frac{n+1}{2}} \\
& \geq \frac{2 K_{1}^{2}}{(n+1)} \sum_{p=1}^{k+1}(p-1)^{n-\frac{1}{2}} \\
& \geq \frac{4 K_{1}^{2}}{(n+1)(2 n+1)} k^{n+1 / 2}
\end{aligned}
$$

and

$$
\begin{aligned}
m=r_{n}(0)+r_{n}(1)+\cdots+r_{n}(k)+r_{n}(k+1) & \leq K_{2}\left(1+\sum_{l=1}^{k+1} l^{\frac{n}{2}-1}\right) \\
& \leq \frac{2 K_{2}}{n}\left[\frac{n}{2}+(k+2)^{\frac{n}{2}}\right] .
\end{aligned}
$$

Hence

$m^{-2} \sum_{i=1}^{m-1} \sum_{j=i+1}^{m}\left\|c_{j}-c_{i}\right\| \geq \frac{K_{1}^{2} n^{2} k^{n+1 / 2}}{K_{2}^{2}(n+1)(2 n+1)(k+2)^{n}}\left(1+\frac{n}{2(k+2)^{\frac{n}{2}}}\right)^{-2}$.

Putting $K_{3}:=\frac{K_{1}^{2} n^{2} 2^{n+2}}{K_{2}^{2}(n+1)(2 n+1) 3^{n}\left(n+2^{\frac{n}{2}+1}\right)^{2}}$, we deduce

$$
m^{-2} \sum_{i=1}^{m-1} \sum_{j=i+1}^{m}\left\|c_{j}-c_{i}\right\| \geq K_{3} \sqrt{k}
$$

It is easy to check that the above computation is still valid when $m$ lies strictly between $r_{n}(0)+r_{n}(1)+\cdots+r_{n}(k)$ and $r_{n}(0)+r_{n}(1)+\cdots+r_{n}(k+1)$. Therefore $\lim _{m \rightarrow+\infty} \frac{1}{m^{2}} \sum_{i=1}^{m-1} \sum_{j=i+1}^{m}\left\|c_{j}-c_{i}\right\|=+\infty$. Let us observe that this minimal averaged growth to infinity is in " $\sqrt{k}$ ", which is extremely slow as compared to the growth of $m$ to infinity.

Let us now compute a lower bound of the sum $m^{-2} \sum_{j=1}^{m}\left\|c_{j}\right\|$. Take for $R$ the square root of an integer, say $R=\sqrt{t}, t \geq 2$. Let us consider that $m$ is equal to $m=r_{n}(0)+r_{n}(1)+\cdots+r_{n}(k+1)$ and let us write it as: $m=r_{n}(t)+r_{n}(t+1)+\cdots+r_{n}(t+u)+w$ for a certain $u \geq 0$ and 
$0 \leq w<r_{n}(t+u+1)$. Then

$$
\sum_{j=1}^{m}\left\|c_{j}\right\| \geq \sum_{t}^{t+u} r_{n}(l) \sqrt{l} \geq K_{1} \sum_{t}^{t+u} l^{\frac{n-1}{2}} .
$$

As above we will make use of the following classical inequalities: for all positive integers $s$ and $r \geq s+1$ and for any real number $\beta>0, s^{\beta}+(s+1)^{\beta}+$ $\cdots+(r-1)^{\beta} \leq \int_{s}^{r} x^{\beta} d x=\frac{r^{\beta+1}-s^{\beta+1}}{\beta+1} \leq(s+1)^{\beta}+(s+2)^{\beta}+\cdots+(r-1)^{\beta}+r^{\beta}$. We obtain the following inequalities:

$$
\sum_{j=1}^{m}\left\|c_{j}\right\| \geq \frac{2 K_{1}}{n+1}\left[(t+u)^{\frac{n+1}{2}}-(t-1)^{\frac{n+1}{2}}\right]
$$

and

$$
\begin{aligned}
\frac{2 K_{1}}{n} & {\left[(t+u)^{n / 2}-(t-1)^{n / 2}\right] } \\
& \leq m \\
& \leq r_{n}(t)+r_{n}(t+1)+\cdots+r_{n}(t+u)+r_{n}(t+u+1) \\
& \leq \frac{2 K_{2}}{n}\left[(t+u+2)^{n / 2}-t^{n / 2}\right] .
\end{aligned}
$$

From them we deduce

$$
\begin{aligned}
\frac{1}{m} \sum_{j=1}^{m}\left\|c_{j}\right\| \geq \frac{K_{1} n \sqrt{u}}{K_{2}(n+1)}\left(\left(1+\frac{t}{u}\right)^{\frac{n+1}{2}}-\right. & \left.\left(\frac{t-1}{u}\right)^{\frac{n+1}{2}}\right) \\
& \cdot\left(\left(1+\frac{2+t}{u}\right)^{n / 2}-\left(\frac{t}{u}\right)^{n / 2}\right)^{-1} .
\end{aligned}
$$

Dividing the above inequality by $m$ once again and changing $t$ into $t-1$ and $t$ into $t+2$ in the corresponding factors gives

$$
\begin{aligned}
\frac{1}{m^{2}} \sum_{j=1}^{m}\left\|c_{j}\right\| \geq \frac{K_{1} n^{2} u^{\frac{1-n}{2}}}{2 K_{2}^{2}(n+1)}\left(\left(1+\frac{(t-1)}{u}\right)^{\frac{n+1}{2}}-\left(\frac{t-1}{u}\right)^{\frac{n+1}{2}}\right) \\
\cdot\left(\left(1+\frac{2+t}{u}\right)^{n / 2}-\left(\frac{2+t}{u}\right)^{n / 2}\right)^{-2}
\end{aligned}
$$

so that, using first-order developments in $(t-1) u^{-1}$, resp. in $(2+t) u^{-1}$, for $u^{-1}$ close to zero, we obtain

$$
\frac{1}{m^{2}} \sum_{j=1}^{m}\left\|c_{j}\right\| \geq \frac{K_{1}(t-1)^{\frac{n-1}{2}}}{K_{2}^{2}} \frac{1}{u(u+2+t)^{n-2}} .
$$

This lower bound, as a function of $u$ on $[1,+\infty)$, goes to zero at infinity. 
Let us now compute a lower bound of the sum $m^{-2} \sum_{j=1}^{m}\left\|c_{j}\right\|+$ $m^{-2} \sum_{1 \leq i<j \leq m}\left\|c_{i}-c_{j}\right\|$. The lower bound given by (A.5) is a function of $k$ and that given by (A.6) a function of $u$. In order to study their sum, we will deduce from the above a relation between $u$ and $\sqrt{k}$ and replace $\sqrt{k}$ by a lower bound of $\sqrt{k}$ in (A.5) which will only depend upon $u$. From the above, with $m=r_{n}(0)+r_{n}(1)+\cdots+r_{n}(k+1)$, the following inequalities hold

$$
\frac{2 K_{1}}{n}\left[(t+u)^{n / 2}-(t-1)^{n / 2}\right] \leq m \leq \frac{2 K_{2}}{n}\left[\frac{n}{2}+(k+2)^{\frac{n}{2}}\right] .
$$

Let $h(x)=(t+x)^{n / 2}$. Then $h(u)-h(-1)=(u+1) h^{\prime}(\xi)$ for a certain $\xi \in[-1, u]$. We deduce $h(u)-h(-1) \geq \frac{n}{2} u(t-1)^{\frac{n}{2}-1}$ since the derivative $h^{\prime}(x)$ is increasing on the interval $[-1, u]$. This last inequality and (A.7) imply

$$
u^{1 / n}\left[\left(\frac{n}{2}\left(\frac{K_{1}}{K_{2}}(t-1)^{\frac{n}{2}-1}-1\right)\right)^{2 / n}-2\right]^{1 / 2} \leq \sqrt{k}
$$

for all $k \geq 1, u \geq 1, t \geq 2$.

Define

$$
g(t, u):=C_{1}(t) \frac{1}{u(u+2+t)^{n-2}}+C_{2}(t) u^{1 / n}
$$

where

$$
C_{1}(t)=K_{1} K_{2}^{-2}(t-1)^{\frac{n-1}{2}}
$$

and

$$
C_{2}(t)=K_{3}\left[\left(\frac{n}{2}\left(\frac{K_{1}}{K_{2}}(t-1)^{\frac{n}{2}-1}-1\right)\right)^{2 / n}-2\right]^{1 / 2} .
$$

From (A.5) in which $\sqrt{k}$ is replaced by the above lower bound and from (A.6), we deduce

$$
\eta(\sqrt{t}, m) \geq g(t, u) .
$$

It is routine to compute the value $u_{\min }(t)$ at which the function $u \rightarrow g(t, u)$ is minimal and the value $g\left(t, u_{\min }(t)\right)$ of its minimum. The equation satisfied by $u_{\min }(t)$ is $n C_{1}(t)(u+2+t)^{1-n}[(n-1) u+2+t]=C_{2}(t) u^{1+1 / n}$ and

$$
\text { (A.11) } g\left(t, u_{\min }(t)\right)=C_{2}(t)\left[\frac{1}{n} \frac{u_{\min }(t)+2+t}{(n-1) u_{\min }(t)+2+t}+1\right]\left(u_{\min }(t)\right)^{1 / n}
$$

Since obviously $u_{\min }(t) \geq 1, \frac{1}{n} \frac{u+2+t}{(n-1) u+2+t}+1 \geq \frac{1}{n(n-1)}+1$ for $t \geq 2, u \geq 1$ and $\lim _{t \rightarrow+\infty} C_{2}(t)=+\infty$, we obtain: $\lim _{t \rightarrow+\infty} g\left(t, u_{\min }(t)\right)=+\infty$. We deduce that for any integer $m$ of the form $r_{n}(0)+r_{n}(1)+\cdots+r_{n}(k+1)$

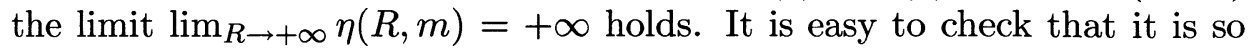


even when $m$ is an arbitrary integer which is not of this form. This implies, after (A.2), that $\lim _{R \rightarrow+\infty} \delta_{2}\left(\mathbb{Z}^{n}, \mathbb{Z}_{R}^{n}\right)=0$, for all $n \geq 5$.

Step 2.- We will make use of the results of Step 1 and of the following three Lemmas. The assumption $n \geq 5$ holds. Let us fix the notations: if $\Gamma$ is a $\mathcal{U D}$-set which contains the origin, then, for all $k \in \mathbb{N}$, denote $\Gamma^{(k)}:=\{x \in \Gamma \mid \sqrt{k} \leq\|x\|<\sqrt{k+1}\}, r_{\Gamma}(\sqrt{k})$ the number of elements of $\Gamma^{(k)}$ and $s(\sqrt{k}):=\max _{\Gamma \in \mathcal{U} \mathcal{D}}\left\{r_{\Gamma}(\sqrt{k})\right\}<\infty$. Since all the functions $\Gamma \rightarrow r_{\Gamma}(\sqrt{k}), k \in \mathbb{N}$, on $\mathcal{U D}$ are valued in $\mathbb{N}$, the maximum $s(\sqrt{k})$ is reached. Since, in particular, $r_{\mathbb{Z}^{n}}(\sqrt{k})=r_{n}(k)$, for any positive integer $k$, the following Lemma is obvious.

Lemma A.1. For any positive integer $k$ the inequality $s(\sqrt{k}) \geq r_{n}(k)$ holds.

In the following, we will enumerate the elements $x_{i}$ of a $\mathcal{U D}$-set $\Lambda$ in such a way that $\left\|x_{j}\right\| \geq\left\|x_{i}\right\|$ as soon as $j \geq i \geq 1$ (with $x_{1}=0$ if $\Lambda$ contains the origin). The following Lemmas show that the sequence $\{s(\sqrt{k}) \mid k \in \mathbb{N}\}$ is universal for splitting up any $\mathcal{U D}$-set into layers of points with the objective of making use of Stolarsky's inequality (Proposition A.2) in a suitable way.

Lemma A.2. Let $\Lambda$ be an infinite $\mathcal{U D}$-set which contains the origin. For all positive integers $M, m \in \mathbb{N}$ such that $\sum_{k=0}^{M} s(\sqrt{k})<m \leq \sum_{k=0}^{M+1} s(\sqrt{k})$, any point $x_{m} \in \Lambda$ indexed by such an integer $m$ satisfies $\left\|x_{m}\right\| \geq \sqrt{M+1}$.

Proof. This fact comes from the way we have enumerated the elements of $\Lambda$. Obviously, any point $x_{m} \in \Lambda$ indexed by such an integer $m$ is such that $\sum_{k=0}^{M} r_{\Lambda}(\sqrt{k}) \leq \sum_{k=0}^{M} s(\sqrt{k})<m$. By definition of the function $r_{\Lambda}$ we obtain the inequality.

Lemma A.3. Let $\Lambda$ be an infinite $\mathcal{U D}$-set which contains the origin. There exists a subset $\Lambda^{*}$ of $\Lambda$, with $0 \in \Lambda^{*}$, and a surjective mapping $\psi_{\Lambda}: \Lambda \rightarrow \mathbb{Z}^{n}$ such that:

(i) $\psi_{\Lambda}(0)=0,\left\|\psi_{\Lambda}(x)\right\| \leq\|x\|$ for all $x \in \Lambda$;

(ii) for all integers $M, m \in \mathbb{N}$ such that $\sum_{k=0}^{M} s(\sqrt{k})<m \leq \sum_{k=0}^{M+1} s(\sqrt{k})$ the following equalities hold: $\left\|\psi_{\Lambda}\left(x_{m}\right)\right\|=\sqrt{M+1}$ for $x_{m} \in \Lambda \backslash \Lambda^{*}$, $\left\|\psi_{\Lambda}\left(x_{m}\right)\right\|=0$ for $x_{m} \in \Lambda^{*}$;

(iii) the restriction of $\psi_{\Lambda}$ to $\{0\} \cup \Lambda \backslash \Lambda^{*}$ is a bijection from $\{0\} \cup \Lambda \backslash \Lambda^{*}$ to $\mathbb{Z}^{n}$;

(iv) when $\Lambda=\mathbb{Z}^{n}$, then $\Lambda^{*}=\{0\}$ and $\psi_{\Lambda}$ is the identity map up to a reenumeration of the elements of the layer $\left(\mathbb{Z}^{n}\right)^{(k)}$ of $\mathbb{Z}^{n}$ for all $k \in \mathbb{N}$. 
Proof. Let us construct the function $\psi_{\Lambda}$. Denote $s^{(M)}:=\sum_{k=0}^{M} s(\sqrt{k})$ for all $M \in \mathbb{N}$. The following $s(\sqrt{M+1})$-tuple of points:

$$
\begin{array}{r}
\left(x_{s^{(M)}+1}, x_{s^{(M)}+2}, \ldots, x_{s^{(M)}+r_{n}(M+1)}, x_{s^{(M)}+r_{n}(M+1)+1},\right. \\
\left.x_{s^{(M)}+r_{n}(M+1)+2}, \ldots, x_{s^{(M+1)}}\right)
\end{array}
$$

of $\Lambda$ will be splitted up into two parts. Let

$$
\Lambda^{*(M)}=\left\{x_{s^{(M)}+r_{n}(M+1)+1}, x_{s^{(M)}+r_{n}(M+1)+2}, \ldots, x_{s^{(M+1)}}\right\}
$$

and $\Lambda^{*}=\cup_{M \in \mathbb{N}} \Lambda^{*(M)}$. Let us put $\psi_{\Lambda}(z)=0$ for all $z \in \Lambda^{*}$, and, for all $M \in \mathbb{N}$ and for all $i=s^{(M)}+1, s^{(M)}+2, \ldots, s^{(M)}+r_{n}(M+1)$, let us put $\psi_{\Lambda}\left(x_{i}\right) \in S(0, \sqrt{M+1}) \cap \mathbb{Z}^{n}$ such that the restriction of $\psi_{\Lambda}$ to $\Lambda \backslash \Lambda^{*}$ is injective. In other terms, the first $r_{n}(M+1)$ points of the above $s(\sqrt{M+1})$-tuple of points are sent injectively by $\psi_{\Lambda}$ to the $r_{n}(M+1)$ elements of $\mathbb{Z}^{n}$ of norm $\sqrt{M+1}$ which lie on the sphere $S(0, \sqrt{M+1})$, the remaining points $x_{s^{(M)}+r_{n}(M+1)+1}, x_{s^{(M)}+r_{n}(M+1)+2}, \ldots, x_{s^{(M+1)}}$ going to the origin of $\mathbb{Z}^{n}$. There is no uniqueness of such a mapping $\psi_{\Lambda}$ : given $\Lambda^{*}$, any re-enumeration $e$ of the elements of $\mathbb{Z}^{n}$ conserving the norm provides another suitable mapping $e \circ \psi_{\Lambda}: \Lambda \rightarrow \mathbb{Z}^{n}$. Properties (i) to (iv) of $\psi_{\Lambda}$ are easy consequences of its definition.

Let us now consider an infinite $\mathcal{U D}$-set $\Lambda$ which contains the origin and let us continue the proof of Proposition A.1 (if $\Lambda$ does not contain the origin we modify slightly a few points close to the origin for having this property). In a similar way as in Step 1 with (A.2), we are looking for a lower bound of the quantity (with $c_{i}, c_{j} \in \Lambda$ and $\left\|c_{i}\right\| \geq R,\left\|c_{j}\right\| \geq R$ )

$$
\eta_{\Lambda}(R, m):=\frac{1}{2 m}+\frac{1}{m^{2}} \sum_{j=1}^{m}\left\|c_{j}\right\|+\frac{1}{m^{2}} \sum_{1 \leq i<j \leq m}\left\|c_{j}-c_{i}\right\|
$$

as a function of $R$ and $m$. Let us observe that the differences $c_{j}-c_{i}$ belong to the translated $\mathcal{U D}$-sets $\Lambda-c_{i}=\left\{\lambda-c_{i} \mid \lambda \in \Lambda\right\}$ of $\Lambda$ which all contain the origin. Let us now compute a lower bound of $m^{-2} \sum_{1 \leq i<j \leq m}\left\|c_{j}-c_{i}\right\|$. For integers $M, m \in \mathbb{N}$ that satisfy

$$
\sum_{k=0}^{M} s(\sqrt{k})<m \leq \sum_{k=0}^{M+1} s(\sqrt{k})
$$

we deduce the following inequality:

$$
\sum_{j=2}^{m}\left\|c_{j}-c_{1}\right\| \geq \sum_{j=2}^{m}\left\|\psi_{\Lambda-c_{1}}\left(c_{j}\right)\right\| \geq \sum_{l=0}^{M} r_{n}(l) \sqrt{l}
$$

from Lemmas A.1, A.2 and A.3. We now proceed with the other sums $\sum_{j=i+1}^{m}\left\|c_{j}-c_{i}\right\|, i \geq 2$. Let us assume that $m=\sum_{q=0}^{M+1} s(\sqrt{q})$. For all 
$i=1,2, \ldots, s(\sqrt{M})$, the difference $m-i$ is greater than $\sum_{q=0}^{M} s(\sqrt{q})$ and this implies

$$
\sum_{j=i+1}^{m}\left\|c_{j}-c_{i}\right\| \geq \sum_{j=i+1}^{m}\left\|\psi_{\Lambda-c_{i}}\left(c_{j}\right)\right\| \geq \sum_{l=0}^{M} r_{n}(l) \sqrt{l} .
$$

We deduce the inequality

$$
\begin{aligned}
\sum_{i=1}^{s(\sqrt{M+1})} \sum_{j=i+1}^{m}\left\|c_{j}-c_{i}\right\| & \geq s(\sqrt{M+1})\left(\sum_{l=0}^{M} r_{n}(l) \sqrt{l}\right) \\
& \geq r_{n}(M+1)\left(\sum_{l=0}^{M} r_{n}(l) \sqrt{l}\right) .
\end{aligned}
$$

Since for all $i, j$ the inequality $\left\|c_{j}-c_{i}\right\| \geq\left\|\psi_{\Lambda-c_{i}}\left(c_{j}\right)\right\|$ holds and that

$$
\begin{aligned}
\sum_{i=1}^{m-1} \sum_{j=i+1}^{m}\left\|c_{j}-c_{i}\right\|= & \sum_{i=1}^{s(\sqrt{M+1})} \sum_{j=i+1}^{m}\left\|c_{j}-c_{i}\right\| \\
& +\sum_{i=s(\sqrt{M+1})+1}^{s(\sqrt{M+1})+s(\sqrt{M})} \sum_{j=i+1}^{m}\left\|c_{j}-c_{i}\right\|+\ldots \\
& +\sum_{i=s(\sqrt{M+1})+s(\sqrt{M})+\ldots+s(\sqrt{2})+1} \sum_{j=i+1}^{m}\left\|c_{j}-c_{i}\right\|,
\end{aligned}
$$

by reproducing the same computation term by term, we deduce

$$
\begin{aligned}
& \sum_{1 \leq i<j \leq m}\left\|c_{j}-c_{i}\right\| \geq r_{n}(M+1)\left(\sum_{l=0}^{M} r_{n}(l) \sqrt{l}\right) \\
& \quad+r_{n}(M)\left(\sum_{l=0}^{M-1} r_{n}(l) \sqrt{l}\right)+\cdots+r_{n}(2) r_{n}(1)+2^{n}
\end{aligned}
$$

This leads to the same inequality as in (A.5), with $m=\sum_{q=0}^{M+1} s(\sqrt{q})$, except that " $k$ " has to be replaced by " $M$ ". Therefore, we obtain

$$
m^{-2} \sum_{1 \leq i<j \leq m}\left\|c_{j}-c_{i}\right\| \geq K_{3} \sqrt{M}
$$

Let us now compute a lower bound of $m^{-2} \sum_{j=1}^{m}\left\|c_{j}\right\|$. Take $R=\sqrt{t}$ with $t \geq 2$ an integer and consider $m=\sum_{q=0}^{M+1} s(\sqrt{q})$. This lower bound corresponds to a distribution by layers of the points $c_{1}, c_{2}, \ldots, c_{m}$ on $\Lambda$ so that they are located as close as possible to the sphere $S(0, R)$. Let us write 
$m$ as the following sum: $m=s(\sqrt{t})+s(\sqrt{t+1})+\cdots+s(\sqrt{t+U})+W$ for certain integers $U \geq 0$ and $0<W \leq s(\sqrt{T t+U+1})$. Then, by Lemma A.3,

$$
\sum_{j=1}^{m}\left\|c_{j}\right\| \geq \sum_{j=1}^{m}\left\|\psi_{\Lambda}\left(c_{j}\right)\right\| \geq \sum_{l=t}^{t+U} r_{n}(l) \sqrt{l} .
$$

Hence, by the same type of computation as in Step 1, and by replacing only " $u$ " by " $U$ ", we deduce

$$
\frac{1}{m^{2}} \sum_{j=1}^{m}\left\|c_{j}\right\| \geq \frac{K_{1}(t-1)^{\frac{n-1}{2}}}{K_{2}^{2}} \frac{1}{U(U+2+t)^{n-2}} .
$$

In order to compute a lower bound of the sum $m^{-2} \sum_{j=1}^{m}\left\|c_{j}\right\|+$ $m^{-2} \sum_{1 \leq i<j \leq m}\left\|c_{j}-c_{i}\right\|$ as a function of $U$ only from (A.12) and (A.13), it remains to give explicitely a relation between $M$ and $U$. This relation comes from the computation of a lower bound of $m$ which will be a function of $M$ only and an upper bound of $m$ which will be a function of $U$ only. Let us compute these bounds. First, since $\sum_{k=0}^{M+1} r_{n}(k) \leq \sum_{k=0}^{M+1} s(\sqrt{k})=m$ we deduce, by the same type of computation as in Step 1 (with " $U$ " instead of " $u$ "),

$$
\frac{2 K_{1}}{n}\left[(t+U)^{n / 2}-(t-1)^{n / 2}\right] \leq \sum_{q=0}^{M+1} s(\sqrt{q})=m .
$$

Second, if $\operatorname{vol}(B(0, x))$ denotes the volume of the ball $B(0, x)$, by counting the maximal possible number of points in $\{x \mid \sqrt{k} \leq\|x\|<\sqrt{k+1}\}$ (in this annulus any point should be at a distance from another one greater than unity), we deduce that the term $s(\sqrt{k}), k \geq 1$, is smaller than

$$
\left(\operatorname{vol}\left(B\left(0, \sqrt{k+1}+\frac{1}{2}\right)\right)-\operatorname{vol}\left(B\left(0, \sqrt{k}-\frac{1}{2}\right)\right)\right)\left(\operatorname{vol}\left(B\left(0, \frac{1}{2}\right)\right)\right)^{-1} .
$$

Therefore

$$
m=\sum_{k=0}^{M+1} s(\sqrt{k}) \leq 1+2^{n} \sum_{k=1}^{M+1}\left[\left(\sqrt{k+1}+\frac{1}{2}\right)^{n}-\left(\sqrt{k}-\frac{1}{2}\right)^{n}\right] .
$$

By a first-order development of each term, we deduce

$$
m \leq 1+n 2^{n} \sum_{k=1}^{M+1}[\sqrt{k+1}-\sqrt{k}+1]\left(\sqrt{k+1}+\frac{1}{2}\right)^{n-1} .
$$

Since $\sqrt{k+1}-\sqrt{k}+1 \leq 2$ we obtain that $m$ is certainly exceeded by $n 2^{n+1} \sum_{k=1}^{M+1}\left(\sqrt{k+1}+\frac{1}{2}\right)^{n-1}$. Now, for all $1 \leq k \leq M+1$, we have 
$\sqrt{k+1}+\frac{1}{2} \leq \sqrt{k+3 \sqrt{M+1}}$. We deduce

$$
\begin{aligned}
m & \leq n 2^{n+1} \sum_{k=1}^{M+1}(k+3 \sqrt{M+1})^{\frac{n-1}{2}} \\
& \leq \frac{n 2^{n+2}}{n+1}\left[(M+2+3 \sqrt{M+1})^{\frac{n+1}{2}}-(1+3 \sqrt{M+1})^{\frac{n+1}{2}}\right] .
\end{aligned}
$$

Denote $l(x)=\left(x+\frac{1+3 \sqrt{M+1}}{M+1}\right)^{\frac{n+1}{2}}$ and $\omega=\sup _{M \geq 1}\left(\sup _{x \in[0,1]} l^{\prime}(x)\right)$. Then it is easy to check, by factorizing $(M+1)^{(n+1) / 2}$ and applying a first-order development to the factors in the right-hand side term of the last inequality that this term is smaller than $n 2^{n+2} \omega(n+1)^{-1}(M+1)^{\frac{n+1}{2}}$. Hence

$$
m \leq n 2^{n+2} \omega(M+1)^{\frac{n+1}{2}} .
$$

From (A.14) and (A.15) (as for (A.7) and (A.8)) we deduce the following inequality

$$
U^{\frac{1}{n+1}}\left[\frac{1}{4}\left(\frac{K_{1}}{2 \omega}\right)^{2 /(n+1)}(t-1)^{\frac{n-2}{n+1}}-1\right]^{1 / 2} \leq \sqrt{M}
$$

Define

$$
g_{\Lambda}(t, U):=\frac{C_{1}(t)}{U(U+2+t)^{n-2}}+C_{3}(t) U^{\frac{1}{n+1}}
$$

where $C_{3}(t):=K_{3}\left[\frac{1}{4}\left(\frac{K_{1}}{2 \omega}\right)^{\frac{2}{n+1}}(t-1)^{\frac{n-2}{n+1}}-1\right]^{1 / 2}$. Then (as in Step 1)

$$
\eta_{\Lambda}(\sqrt{t}, m) \geq g_{\Lambda}\left(t, U_{\min }(t)\right),
$$

for all $m=\sum_{k=0}^{M+1} s(\sqrt{k})$, where $U_{\min }(t)$ is the value at which the function $U \rightarrow g_{\Lambda}(t, U)$ is minimal. The proof of $\lim _{t \rightarrow+\infty} g_{\Lambda}\left(t, U_{\min }(t)\right)=+\infty$ is similar as in Step 1, for any integer $m$. This implies, after (A.2), that $\lim _{R \rightarrow+\infty} \delta_{2}\left(\Lambda, \Lambda_{R}\right)=0$ for all $\mathcal{U D}$-set $\Lambda$ and all $n \geq 5$. This convergence is obviously uniform in the sense stated in Proposition A.1 since the sequence $(s(\sqrt{k}))_{k}$ is universal and optimal for splitting up any $\mathcal{U} \mathcal{D}$-set $\Lambda$.

Step 3.- If $\Lambda$ is a $\mathcal{U D}$-set in $\mathbb{R}^{n}$ with $n \leq 4$, it can be viewed as a $\mathcal{U D}$-set in $\mathbb{R}^{5}$. Since Proposition A.1 is true for $n=5$ by Step 2, it is also true in lower dimensions by descent.

Acknowledgements. The authors thank the anonymous referee for very helpful comments and suggestions. 


\section{References}

[BL] M. BAAKE, D. LENZ, Dynamical systems on translation bounded measures: pure point dynamical and diffraction spectra. Ergod. Th. \& Dynam. Sys. 24 (6) (2004), 1867-1893.

[B] N. Bourbaki, General Topology. Chapter IX, Addison-Wesley, Reading, 1966.

[Bo] L. BowEN, On the existence of completely saturated packings and completely reduced coverings. Geom. Dedicata 98 (2003), 211-226.

[Ca] J.W.S. CASSELS, An introduction to the Geometry of Numbers. Springer Verlag, 1959.

[Ch] C. Chabauty, Limite d'Ensembles et Géométrie des Nombres. Bull. Soc. Math. Fr. 78 (1950), 143-151.

[CS] J.H. CoNWAY, N.J.A. SlOANE, Sphere packings, lattices and groups. Springer-Verlag, Berlin, 1999, third edition.

[Dw] S. Dworkin, Spectral Theory and X-Ray Diffraction. J. Math. Phys. 34 (7) (1993), 2965-2967.

[GVG] J.-P. Gazeau, J.-L. Verger-Gaugry, Geometric study of the beta-integers for a Perron number and mathematical quasicrystals. J. Théorie Nombres Bordeaux 16 (2004), 125-149.

[Go] J.-B. GoUÉRÉ, Quasicrystals and almost-periodicity. Comm. Math. Phys. (2005), accepted.

[Groe] H. Groemer, Continuity properties of Voronoi domains. Monatsh. Math. 75 (1971), 423-431.

[Gr] E. Grosswald, Representations of Integers as Sums of Squares. Springer-Verlag, New York, 1985.

[GL] P.M. GRUBer, C.G. LeKKERKERKER, Geometry of Numbers. North-Holland, 1987.

[Ha] T.C. HALES, Sphere Packings I. Discrete Comput. Geom. 17 (1997), 1-51.

[Ke] J.L. Kelley, Hyperspaces of a Continuum. Trans. Amer. Math. Soc. 52 (1942), 22-36.

[La] J.C. LAGARIAS, Bounds for Local Density of Sphere Packings and the Kepler Conjecture. Discrete Comput. Geom. 27 (2002), 165-193.

[Ma] K. MAHLER, On Lattice Points in n-dimensional Star Bodies.I. Existence Theorems. Proc. Roy. Soc. London A 187 (1946), 151-187.

[MS] A.M. MACBEATH, S. SWIERCZKOWSKI, Limits of lattices in a compactly generated group. Canad. J. Math. 12 (1960), 427-437.

[Mf] R.B. MCFEAT, Geometry of numbers in adele spaces. Dissertationes Math. (Rozprawy mat.), Warsawa, 88 (1971), 1-49.

[Mi] E. Michael, Topologies on Spaces of Subsets. Trans. Amer. Math. Soc. 71 (1951), $152-182$.

[Mo] R.V. Moody, Meyer sets and their duals. In The Mathematics of Long-Range Aperiodic Order, Ed. by R.V. Moody, Kluwer Academic Publishers (1997), 403-441.

[Mu] D. Mumford, A Remark on Mahler's Compactness Theorem. Proc. of the Amer. Math. Soc. 28 (1971), 289-294.

[MVG] G. MurAz, J.-L. Verger-Gaugry, On lower bounds of the density of Delone sets and holes in sequences of sphere packings. Exp. Math. 14:1 (2005), 49-59.

[MVG1] G. MURAz, J.-L. Verger-GAUGRY, On continuity properties of Voronoi domains and a theorem of Groemer. Preprint (2004).

[RW] C. RADIN, M. WolfF, Space Tilings and Local Isomorphism. Geom. Dedicata 42 (1992), 355-360.

[Ro] E. Arthur Robinson, JR., The Dynamical Theory of Tilings and Quasicrystallography. In Ergodic Theory of $\mathbb{Z}^{d}$-actions, (Warwick 1993-4), London Math. Soc. Lec. Note Ser. 228, Cambridge Univ. Press, Cambridge, 451-473.

[Ro] C.A. Rogers, Packing and Covering. Cambridge University Press, 1964.

[RSD] K. ROGERS, H.P.F. SWINNERTON-DYER, The Geometry of Numbers over algebraic number fields. Trans. Amer. Math. Soc. 88 (1958), 227-242.

[So] B. Solomyak, Spectrum of Dynamical Systems Arising from Delone Sets. In Quasicrystals and Discrete Geometry, Fields Institute Monographs, J. Patera Ed., 10 (1998), AMS, 265-275. 
[St] K.B. Stolarsky, Sums of distances between points on a sphere. Proc. Amer. Math. Soc. 35 (1972), 547-549.

[We] A. WEIL, Sur les Espaces à Structure Uniforme et sur la Topologie Générale. Hermann, Paris, 1938.

Gilbert MURAZ \& Jean-Louis Verger-Gaugry

Institut Fourier - CNRS UMR 5582

Université de Grenoble I

BP 74 - Domaine Universitaire

38402 Saint Martin d'Hères, France

E-mail : Gilbert.Muraz@ujf-grenoble.fr

E-mail : jlverger@ujf-grenoble.fr

$U R L:$ http://www-fourier.ujf-grenoble.fr 